\title{
Detection of real periodicity in the terrestrial impact crater record: quantity and quality requirements
}

\author{
J. Lyytinen, L. Jetsu, P. Kajatkari, and S. Porceddu
}

Observatory, PO Box 14, 00014 University of Helsinki, Finland

e-mail: joonas.lyytinen@iki.fi

Received 22 October 2008 / Accepted 30 January 2009

\section{ABSTRACT}

\begin{abstract}
Aims. To determine the quantity and quality requirements for the terrestrial impact crater data which would allow reliable detection of real periodicity.

Methods. Artificial impact crater data samples of different size and accuracy are simulated. Erosion is considered, as well as the effect of the unknown ratio between periodic and aperiodic impacts. The probabilities for detecting real and false periodicities are solved with the Rayleigh test from these simulated data.

Results. Reliable detection of real periodicity is currently impossible - unless all impacts on Earth have been periodic.
\end{abstract}

Key words. Earth - minor planets, asteroids - Oort cloud - methods: statistical - Galaxy: solar neighborhood - comets: general

\section{Introduction}

Alvarez \& Muller (1984) detected a 28.4 Myr periodicity in the ages of terrestrial impact craters. In the same volume of Nature, two theories on the possible astronomical process capable of triggering periodic comet showers from the Oort cloud were presented. Davis et al. (1984) and Whitmire \& Jackson (1984) suggested that an unseen solar companion "Nemesis" causes periodic perturbances to the Oort cloud, and Rampino \& Stothers (1984) and Schwartz \& James (1984) argued for perturbances caused by the oscillation of the Solar System through the Galactic plane. For a quarter of a century, evidence that confirms the existence of real periodicity in these catastrophic events has been published, e.g. by Stothers (1998), Moon et al. (2003), Yabushita (2004), Chang \& Moon (2005), Stothers (2006) and Wickramasinghe \& Napier (2008). Evidence against such periodicity has also been presented, e.g. by Bailey \& Stagg (1988), Grieve et al. (1988), Deutsch \& Schaerer (1994), Grieve \& Pesonen (1996), Jetsu (1997) and Jetsu \& Pelt (2000).

We decided to determine the quantity and quality requirements for the terrestrial impact crater data that would allow reliable detection of real periodicity, if present. We also noted the presence of erosion and different values for the ratio between periodic and aperiodic impacts.

\section{Real data}

\subsection{Detection rate}

Several geological processes reduce the detectability of terrestrial impact craters as a function of time. This decrease in detectability was estimated from the crater data in The Earth Impact Database ${ }^{1}$ maintained by the Planetary and Space Science Centre at the University of New Brunswick. These data

\footnotetext{
1 Planetary and Space Science Centre. The Earth Impact Database, http://www . unb.ca/passc/ImpactDatabase/ (accessed: 11 Aug. 2008).
}

contained $n=174$ impact structures. The errors for the ages were available for $n=89$ craters. Simultaneous pairs of events were combined as in Jetsu (1997). As in that particular study, the age $t_{i} \pm \sigma_{i}[\mathrm{Myr}]$ and the diameter $D_{i}[\mathrm{~km}]$ of each crater were then used for selecting the two subsamples of data:

$C_{2}: t_{i} \leq 250, \sigma_{i} \leq 20, D \geq 5(n=33)$

$C_{3}: 5 \leq t_{i} \leq 300, \sigma_{i} \leq 20(n=40)$.

These data are given in Table 1 . It contains the following information for each crater: name (Col. 1), location (Col. 2), age (Col. 3), diameter (Col. 4), belongs ("Yes") or does not belong ("No") to the sub-sample $C_{2}$ or $C_{3}$ (Cols. 5 and 6), and the cycle number $k_{i}=1,2, \ldots, 10$ in $C_{2}$ and $C_{3}$ (Cols. 7 and 8). For each $t_{i}$, this cycle number is $k_{i}=1+\operatorname{INT}\left[K\left(t_{i}-t_{1}\right) /\left(t_{n}-t_{1}\right)\right]$, where INT $[x]$ removes the decimal part of its argument $x$ and $K=10$. In other words, the data in $C_{2}$ and $C_{3}$ were divided into ten subintervals of equal length $\left(t_{n}-t_{1}\right) / 10$ and the value of $k_{i}$ indicates the particular subinterval where each $t_{i}$ belongs. The $k_{i}$ values were used for calculating the fractional estimates $A_{k}$ for the detectability of the craters as a function of their age. The results are given in Table 2. First, the number of craters within each cycle $k$ was divided by the total number of craters in samples $C_{2}$ and $C_{3}$ (Table 2: Cols. 2 and 3). Because these estimates fluctuated considerably, the averages for each pair of consecutive cycles were then calculated (Table 2: Cols. 4 and 5). The final values for the fractional detectability estimates $A_{k}$ were fixed to the mean of both samples (Table 2: Col. 6). Note that these fractional estimates satisfy the relation $\sum_{k=1}^{K=10} A_{k}=1$.

\subsection{Age uncertainty}

For $K$ cycles of equal length over the whole time span of the data $t_{1} \pm \sigma_{1}, t_{2} \pm \sigma_{2}, \ldots, t_{n} \pm \sigma_{n}$, the period is $P_{K}=\left(t_{n}-t_{1}\right) / K$. Our definition for the average error of these data is

$\sigma_{P^{\prime}}=\frac{1}{n} \sum_{i=1}^{n} \frac{\sigma_{i}}{P_{K}}$, 
Table 1. The most recent terrestrial impact crater data obtained in August 11th, 2008 from The Earth Impact Database. The combined simultaneous pairs of impacts are marked with " $\star$ ". The following information of these craters is given: name (Col. 1), location (Col. 2), age and its error (Col. 3: $\left[t_{i} \pm \sigma_{i}\right]=\mathrm{My}$ ), diameter (Col. 4: $\left[D_{i}\right]=\mathrm{km}$ ) and the crater belongs ("Yes") or does not belong ("No") to the sub-sample $C_{2}$ or $C_{3}($ Cols. 5 and 6). The value of $k_{i}$ is the cycle number in $C_{2}$ and $C_{3}$ (Cols. 7 and 8).

\begin{tabular}{|c|c|c|c|c|c|c|c|}
\hline Crater & Location & $\overline{t_{i} \pm \sigma_{i}}$ & $\overline{\overline{D_{i}}}$ & $C_{2}$ & $\overline{C_{3}}$ & $\overline{k_{i} \text { in } C_{2}}$ & $\overline{k_{i} \text { in } C_{3}}$ \\
\hline Zhamanshin & Kazakhstan & $0.9 \pm 0.1$ & 14 & Yes & No & 1 & $\overline{-}$ \\
\hline El'gygytgyn & Russian & $3.5 \pm 0.5$ & 18 & Yes & No & 1 & - \\
\hline Bigach/Karla $\star$ & Kazakhstan/Russia & $5.0 \pm 0.7$ & 12.8 & Yes & Yes & 1 & 1 \\
\hline Steinheim & Germany & $15 \pm 1$ & 3.8 & No & Yes & - & 1 \\
\hline Ries & Germany & $15.1 \pm 0.1$ & 24 & Yes & Yes & 1 & 1 \\
\hline Chesapeake Bay & USA & $35.5 \pm 0.3$ & 90 & Yes & Yes & 2 & 2 \\
\hline Popigai & Russia & $35.7 \pm 0.2$ & 100 & Yes & Yes & 2 & 2 \\
\hline Mistastin & Canada & $36.4 \pm 4.0$ & 28 & Yes & Yes & 2 & 2 \\
\hline Wanapitei & Canada & $37.2 \pm 1.2$ & 7.5 & Yes & Yes & 2 & 2 \\
\hline Beyenchime-Salaatin/Logancha & Russia/Russia & $40 \pm 14$ & 21.5 & Yes & Yes & 2 & 2 \\
\hline Logoisk & Belarus & $42.3 \pm 1.1$ & 15 & Yes & Yes & 2 & 2 \\
\hline Shunak & Kazakhstan & $45 \pm 10$ & 2.8 & No & Yes & - & 2 \\
\hline Chiyli/Ragozinka $\star$ & Kazakhstan/Russia & $46.0 \pm 2.1$ & 10.5 & Yes & Yes & 2 & 2 \\
\hline Gusev/Kamensk ^ & Russia/Russia & $49.00 \pm 0.14$ & 25.2 & Yes & Yes & 2 & 2 \\
\hline Montagnais & Canada & $50.50 \pm 0.76$ & 45 & Yes & Yes & 3 & 2 \\
\hline Marquez & USA & $58 \pm 2$ & 12.7 & Yes & Yes & 3 & 2 \\
\hline Chicxulub & Mexico & $64.98 \pm 0.05$ & 170 & Yes & Yes & 3 & 3 \\
\hline Boltysh & Ukraine & $65.17 \pm 0.64$ & 24 & Yes & Yes & 3 & 3 \\
\hline Kara & Russia & $70.3 \pm 2.2$ & 65 & Yes & Yes & 3 & 3 \\
\hline Lappajärvi & Finland & $73.3 \pm 5.3$ & 23 & Yes & Yes & 3 & 3 \\
\hline Manson & USA & $73.8 \pm 0.3$ & 35 & Yes & Yes & 3 & 3 \\
\hline Zeleny Gai & Ukraine & $80 \pm 20$ & 3.5 & No & Yes & - & 3 \\
\hline Wetumpka & USA & $81.0 \pm 1.5$ & 6.5 & Yes & Yes & 4 & 3 \\
\hline Dellen & Sweden & $89.0 \pm 2.7$ & 19 & Yes & Yes & 4 & 3 \\
\hline Steen River & Canada & $91 \pm 7$ & 25 & Yes & Yes & 4 & 4 \\
\hline Deep Bay & Canada & $99 \pm 4$ & 13 & Yes & Yes & 5 & 4 \\
\hline Carswell & Canada & $115 \pm 10$ & 39 & Yes & Yes & 5 & 4 \\
\hline Rotmistrovka & Ukraine & $120 \pm 10$ & 2.7 & No & Yes & - & 5 \\
\hline Mien & Sweden & $121.0 \pm 2.3$ & 9 & Yes & Yes & 5 & 5 \\
\hline Tookoonooka & Australia & $128 \pm 5$ & 55 & Yes & Yes & 6 & 5 \\
\hline Mjølnir & Norway & $142.0 \pm 2.6$ & 40 & Yes & Yes & 6 & 5 \\
\hline Gosses Bluff & Australia & $142.5 \pm 0.8$ & 22 & Yes & Yes & 6 & 5 \\
\hline Morokweng & South Africa & $145.0 \pm 0.8$ & 70 & Yes & Yes & 6 & 5 \\
\hline Tabun-Khara-Obo & Mongolia & $150 \pm 20$ & 1.3 & No & Yes & - & 6 \\
\hline Zapadnaya & Ukraine & $165 \pm 5$ & 3.2 & No & Yes & - & 6 \\
\hline Puchezh-Katunki & Russia & $167 \pm 3$ & 80 & Yes & Yes & 7 & 6 \\
\hline Obolon“ & Ukraine & $169 \pm 7$ & 20 & Yes & Yes & 7 & 6 \\
\hline Viewfield & Canada & $190 \pm 20$ & 2.5 & No & Yes & - & 7 \\
\hline Rochechouart/Manicouagan $\star$ & France/Canada & $214.0 \pm 0.7$ & 103 & Yes & Yes & 9 & 8 \\
\hline Araguainha & Brazil & $244.40 \pm 3.25$ & 40 & Yes & Yes & 10 & 9 \\
\hline Ternovka & Ukraine & $280 \pm 10$ & 10 & No & Yes & - & 10 \\
\hline Clearwater East/West $\star$ & Canada/Canada & $290 \pm 14$ & 43.6 & No & Yes & - & 10 \\
\hline
\end{tabular}

Table 2. The fractional crater detectability estimates calculated from data of Table 1: Col. $1(k)$ is the cycle number. Columns $2\left(a_{1}\right)$ and $3\left(a_{2}\right)$ give the number of craters within cycle $k$ divided by the total number of craters in samples $C_{2}(n=33)$ and $C_{3}(n=40)$. Columns $4\left(a_{3}\right)$ and 5 $\left(a_{4}\right)$ give the averages for pairs of two consecutive cycles in samples $C_{2}$ and $C_{3}$. Column $6\left(A_{k}=\left(a_{3}+a_{4}\right) / 2\right)$ is the mean of both samples.

\begin{tabular}{c|rr|rr|c}
\hline \hline$k$ & $C_{2}: a_{1}$ & $C_{3}: a_{2}$ & $C_{2}: a_{3}$ & $C_{3}: a_{4}$ & $A_{k}=\left(a_{3}+a_{4}\right) / 2$ \\
\hline 1 & $4 / 33$ & $3 / 40$ & $6 / 33$ & $7 / 40$ & $A_{1}=0.1784$ \\
2 & $8 / 33$ & $11 / 40$ & $6 / 33$ & $7 / 40$ & $A_{2}=0.1784$ \\
\hline 3 & $7 / 33$ & $8 / 40$ & $5 / 33$ & $5.5 / 40$ & $A_{3}=0.1445$ \\
4 & $3 / 33$ & $3 / 40$ & $5 / 33$ & $5.5 / 40$ & $A_{4}=0.1445$ \\
\hline 5 & $3 / 33$ & $6 / 40$ & $3.5 / 33$ & $5 / 40$ & $A_{5}=0.1155$ \\
6 & $4 / 33$ & $4 / 40$ & $3.5 / 33$ & $5 / 40$ & $A_{6}=0.1155$ \\
\hline 7 & $2 / 33$ & $1 / 40$ & $1 / 33$ & $1 / 40$ & $A_{7}=0.0277$ \\
8 & $0 / 33$ & $1 / 40$ & $1 / 33$ & $1 / 40$ & $A_{8}=0.0277$ \\
\hline 9 & $1 / 33$ & $1 / 40$ & $1 / 33$ & $1.5 / 40$ & $A_{9}=0.0339$ \\
10 & $1 / 33$ & $2 / 40$ & $1 / 33$ & $1.5 / 40$ & $A_{10}=0.0339$ \\
\hline
\end{tabular}

where $\sigma_{P^{\prime}}$ is measured in the units of $P_{K}$. If the subsamples $C_{2}$ and $C_{3}$ of Table 1 both contained $K=10$ cycles, the respective errors would be $\sigma_{P^{\prime}}=0.11$ and $\sigma_{P^{\prime}}=0.17$. In our simulations, it was assumed that the error of the simulated data has a Gaussian distribution

$$
\begin{aligned}
& f_{\mathrm{G}}\left(t, \mu, \sigma_{P}\right)=\frac{1}{\sigma_{P} \sqrt{2 \pi}} \mathrm{e}^{-\frac{1}{2}\left(\frac{t-\mu}{\sigma_{P}}\right)^{2}} \\
& F_{\mathrm{G}}\left(t, \mu, \sigma_{P}\right)=\frac{1}{\sigma_{P} \sqrt{2 \pi}} \int_{-\infty}^{t} \mathrm{e}^{-\frac{1}{2}\left(\frac{t^{\prime}-\mu}{\sigma_{P}}\right)^{2}} \mathrm{~d} t^{\prime},
\end{aligned}
$$

where $f_{\mathrm{G}}\left(t, \mu, \sigma_{P}\right)$ is the probability density function, $F_{\mathrm{G}}\left(t, \mu, \sigma_{P}\right)$ is the cumulative distribution function and the mean is $\mu=0$. The error $\sigma_{P}$ for the simulated data is measured in the units of the simulated $P$. Note that we calculated the error $\sigma_{P^{\prime}}$ from the real data (Eq. (1)), while the error used for the simulated data was $\sigma_{P}$ (Eqs. (2) and (3)). 


\subsection{Origin of periodicity}

The periodicity detected by Alvarez \& Muller (1984) in the terrestrial impact crater record has been suggested to be caused by regular "comet showers" from the Oort cloud towards the inner solar system (Davis et al. 1984; Whitmire \& Jackson 1984; Rampino \& Stothers 1984; Schwartz \& James 1984). However, direct evidence for such comet showers is lacking. Although Farley (1998) argued for a cometary shower hitting the Earth 35 Myr ago, his findings have been disputed by Tagle \& Claeys (2004).

Since 1984, the proposed periodicities for the comet showers have ranged between 20 and 40 Myr. Hills (1981), among others, has shown that a comet survives less than 1 Myr in the inner Solar System. Each aforementioned comet shower could therefore be considered as "an instantaneous event" in the simulation of the periodic part in Sect. 3.1. Finally, we emphasize that no evidence have been presented for all comet impacts on Earth having been periodic.

\subsection{Origin of aperiodicity}

According to Neukum \& Ivanov (1994), the impact cratering rate of the Earth-Moon system has been nearly constant for approximately 3 billion years. Gladman et al. (1997) have described how collisions in the main-asteroid-belt can eventually transport asteroids to Earth-crossing orbits. Some fragments of these colliding bodies drift into the gravitational resonance areas of the belt, where their orbits decay into planet-crossing orbits. Vokrouhlicky \& Farinella (2000) and Bottke et al. (2002a) have shown how the combination of the Yarkovski effect and the aforementioned collisional dynamics cause a continuous drift of objects from the main-asteroid-belt. Thus the observed Near Earth Object (NEO) population would consist mainly of former main-asteroid-belt objects, but some NEOs might also be ancient comet nuclei. In short, the currently known physical processes indicate that all asteroid impacts on Earth have been aperiodic.

\section{Simulated data}

In this section, we will formulate the simulation of a fully periodic case (Sect. 3.1) and a fully aperiodic case (Sect. 3.2), as well as their combination (Sect. 3.3).

\subsection{Periodic component}

In the fully periodic case we assumed that periodic comet showers have occurred and simulated $K=10$ such showers at the epochs $t_{k}=k-1 / 2$, where $k=1,2, \ldots, K$. It was assumed that the duration of each comet shower is much shorter than the pe$\operatorname{riod} P_{K}=P=1$ and can be approximated with the Dirac delta function. This gave the probability density and the cumulative distribution functions

$$
\begin{aligned}
& f_{P}\left(t, \sigma_{P}\right)=\sum_{k=1}^{K} A_{k} f_{\mathrm{G}}\left(t, t_{k}, \sigma_{P}\right) . \\
& F_{P}\left(t, \sigma_{P}\right)=\sum_{k=1}^{K} A_{k} F_{\mathrm{G}}\left(t, t_{k}, \sigma_{P}\right) .
\end{aligned}
$$

These forms represent the convolution of the Gaussian error (Eqs. (2) and (3)) with a Dirac delta function at each $t_{k}$. The multipliers $A_{k}$ ensured that $\int_{-\infty}^{\infty} f_{P}\left(t, \sigma_{P}\right)=1$. The subscript " $P$ " in $f_{P}(t)$ and $F_{P}(t)$ was adopted to denote the periodic
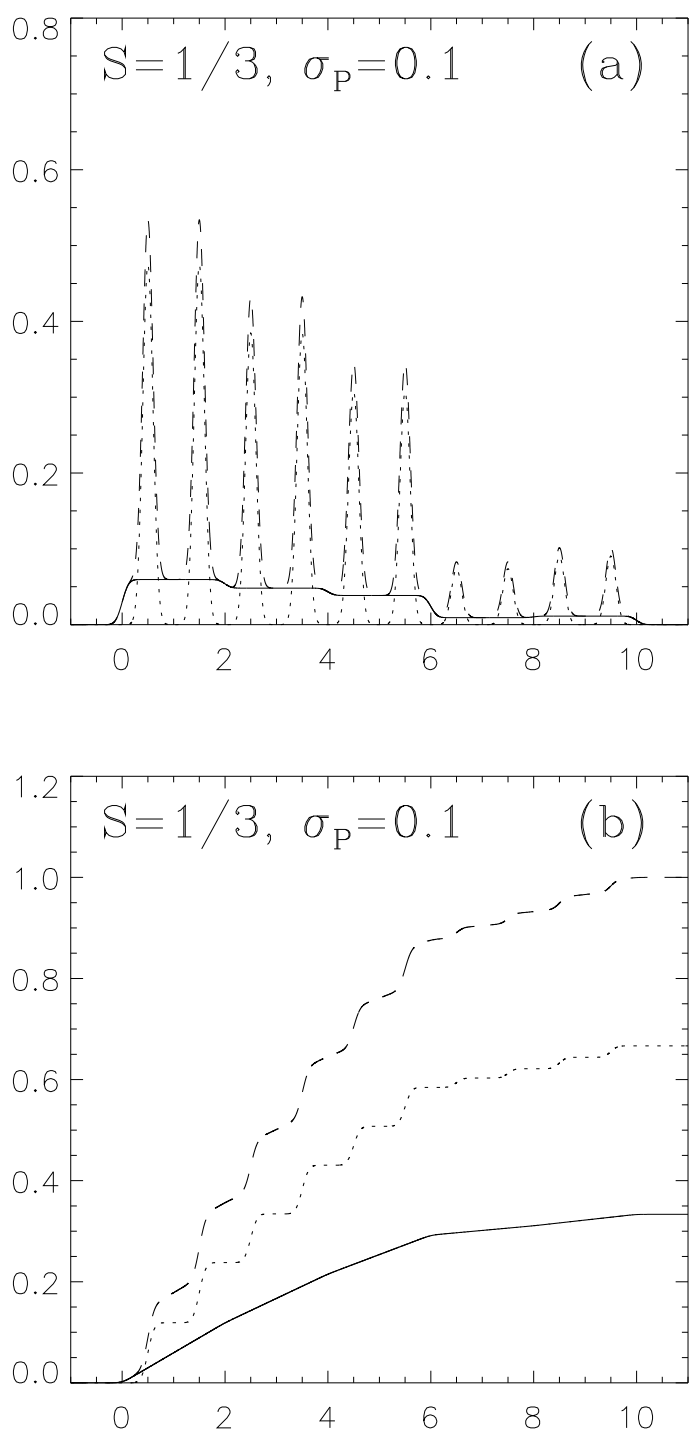

Fig. 1. The simulated case with $S=1 / 3$ and $\sigma_{P}=0.1$ : a) the dashed line denotes the probability density function $f_{\mathrm{AP}}\left(t, S, \sigma_{P}\right)$ of Eq. (8). The continuous line shows the aperiodic component $S f_{\mathrm{A}}\left(t, \sigma_{P}\right)$, while the dotted line shows the periodic component $(1-S) f_{P}\left(t, \sigma_{P}\right)$. b) The respective cumulative distribution functions are $F_{\mathrm{AP}}\left(t, S, \sigma_{P}\right), S F_{\mathrm{A}}\left(t, \sigma_{P}\right)$ and $(1-S) F_{P}\left(t, \sigma_{P}\right)$ of Eq. (9).

component in the simulated crater distribution. One example of these two functions $f_{P}\left(t, \sigma_{P}\right)$ and $F_{P}\left(t, \sigma_{P}\right)$ is shown in Figs. 1a,b (dotted lines), where $\sigma_{P}=0.1$ and both functions have been multiplied with the constant $(1-S)=2 / 3$.

\subsection{Aperiodic component}

In the fully aperiodic case, we first defined the following probability density function

$f_{2}(t)=\sum_{k=1}^{K} A_{k} f_{1}\left(t, a_{k}, b_{k}\right)$

where $a_{k}=k-1, b_{k}=k$ and $f_{1}\left(t, a_{k}, b_{k}\right)= \begin{cases}0, & t<a_{k} \\ \left(b_{k}-a_{k}\right)^{-1}, & a_{k} \leq t<b_{k} \\ 0, & t \geq b_{k} .\end{cases}$

Again, the $A_{k}$ values were used to represent the fraction of craters detected within the $k$ th cycle in this aperiodic case 
(i.e. uniform distribution within each cycle) and to ensure that $\int_{-\infty}^{\infty} f_{\mathrm{A}}\left(t, \sigma_{P}\right)=1$. The cumulative distribution function was

$F_{2}(t)=\sum_{k=1}^{K} A_{k} F_{1}\left(t, a_{k}, b_{k}\right)$,

where $F_{1}\left(t, a_{k}, b_{k}\right)= \begin{cases}0, & t<a_{k} \\ \left(t-a_{k}\right) /\left(b_{k}-a_{k}\right), & a_{k} \leq t<b_{k} \\ 1, & t \geq b_{k} .\end{cases}$

The convolution of $f_{2}(t)$ and $F_{2}(t)$ with the Gaussian error (Eqs. (2) and (3)) gave the following probability density and cumulative distribution functions

$$
\begin{aligned}
f_{\mathrm{A}}\left(t, \sigma_{P}\right)= & \sum_{k=1}^{K} A_{k}\left[F_{\mathrm{G}}\left(t, k-1, \sigma_{P}\right)-F_{\mathrm{G}}\left(t, k, \sigma_{P}\right)\right] \\
F_{\mathrm{A}}\left(t, \sigma_{P}\right)= & \sum_{k=1}^{K} A_{k}\left\{F_{\mathrm{G}}\left(t, k, \sigma_{P}\right)\right. \\
& +(t-k+1)\left[F_{\mathrm{G}}\left(t, k-1, \sigma_{P}\right)-F_{\mathrm{G}}\left(t, k, \sigma_{P}\right)\right] \\
& \left.+\sigma_{P}^{2}\left[f_{\mathrm{G}}\left(t, k-1, \sigma_{P}\right)-f_{\mathrm{G}}\left(t, k, \sigma_{P}\right)\right]\right\},
\end{aligned}
$$

where the subscript "A" in $f_{\mathrm{A}}(t)$ and $F_{\mathrm{A}}(t)$ was adopted to denote the aperiodic component. One example of these two functions $f_{\mathrm{A}}\left(t, \sigma_{P}\right)$ and $F_{\mathrm{A}}\left(t, \sigma_{P}\right)$ is shown in Figs. 1a,b (continuous lines), where $\sigma_{P}=0.1$ and both functions have been multiplied with the constant $S=1 / 3$.

\subsection{Combined components}

Finally, the combination of the periodic and aperiodic cases was simulated with the functions

$$
\begin{aligned}
& f_{\mathrm{AP}}\left(t, S, \sigma_{P}\right)=S f_{\mathrm{A}}\left(t, \sigma_{P}\right)+(1-S) f_{P}\left(t, \sigma_{P}\right) \\
& F_{\mathrm{AP}}\left(t, S, \sigma_{P}\right)=S F_{\mathrm{A}}\left(t, \sigma_{P}\right)+(1-S) F_{P}\left(t, \sigma_{P}\right),
\end{aligned}
$$

where the constant $0 \leq S \leq 1$ modified the relative contribution of each component. The subscript "AP" in $f_{\mathrm{AP}}(t)$ and $F_{\mathrm{AP}}(t)$ was used to denote the combination of periodic and aperiodic cases. These two functions are plotted for the particular case $S=1 / 3$ and $\sigma_{P}=0.1$ in Figs. 1a,b (dashed lines).

In this paper, we have simulated the cases $S=0$ (fully periodic), $S=1 / 3$ or $2 / 3$ (partly periodic) and $S=1$ (fully aperiodic). For all these $S$ values, all combinations of $n=$ $10,25,50,75,100$ or 200 and $\sigma_{P}=0.05,0.1,0.2$ or 0.3 were simulated. The simulation process was simple. First the values of $n, \sigma_{P}$ and $S$ were fixed. Then a series of $x_{1}, x_{2}, . ., x_{n}$ random numbers were generated from an uniform distribution $[0,1)$. Finally, the cumulative distribution function was used to solve the simulated data values $\bar{t}^{*}=\left[t_{1}, t_{2}, . ., t_{n}\right]$, which fulfilled the relation $x_{i}=F_{\mathrm{AP}}\left(t_{i}, \sigma_{P}, S\right)$. Note that these simulated $\bar{t}^{*}$ data and their error $\sigma_{P}$ were both measured in the units of $P=1$.

\section{Rayleigh test}

The Rayleigh test is formulated as in Jetsu \& Pelt (2000). The phases for the time points $t_{1} \leq t_{2} \leq \ldots \leq t_{n}$ are

$\phi_{i}=\operatorname{FRAC}\left[\left(t_{i}-t_{0}\right) P^{-1}\right]=\operatorname{FRAC}\left[\left(t_{i}-t_{0}\right) f\right]$,

where $\operatorname{FRAC}[x]$ removes the integer part of its argument $x, f=$ $P^{-1}$ is the tested frequency and $t_{0}$ is an arbitrary epoch. The "null hypothesis" is
$H_{0}$ : "the phases $\phi_{i}$ calculated with an arbitrary tested period $P$ have a random distribution between $[0,1)$ ".

The Rayleigh test statistic is

$z(f)=\frac{1}{n}\left[\left(\sum_{i=1}^{n} \cos \theta_{i}\right)^{2}+\left(\sum_{i=1}^{n} \sin \theta_{i}\right)^{2}\right]$,

where $\theta_{i}=2 \pi \phi_{i} \equiv 2 \pi f t_{i}$ is the phase angle. Note that $z$ does not depend on the chosen $t_{0}$. Under $H_{0}$, the probability density function is $f(z)=\mathrm{e}^{-z}$, while the cumulative distribution function is $P\left(z \leq z_{0}\right)=F\left(z_{0}\right)=1-\mathrm{e}^{-z_{0}}$ (Kruger et al. 2002). We performed the Rayleigh test between $f_{\min }=P_{\max }^{-1}=0.5$ and $f_{\text {max }}=P_{\text {min }}^{-1}=2.0$, i.e. the periodogram $z(f)$ was calculated within the frequency range $[0.5,2.0]$. The estimate for the number of independent statistical tests was $m=\operatorname{INT}\left[\left(f_{\max }-f_{\min }\right) / f_{0}\right]$, where $f_{0}=\left(t_{n}-t_{1}\right)^{-1}$ was the distance between two independent tested frequencies and INT $[x]$ removed the decimal part of its argument $x$. The probability that $z$ reached $z_{0}$ at least once in all these $m$ independent statistical tests was

$Q=P\left(z \geq z_{0}\right)=1-\left(1-\mathrm{e}^{-z_{0}}\right)^{m}$.

This parameter $Q$ is hereafter called the critical level. The null hypothesis $H_{0}$ was rejected if, and only if

$Q<\gamma$

where $\gamma$ is hereafter called the preassigned significance level. This parameter $\gamma$ expressed the probability of falsely rejecting $H_{0}$ when it was in fact true.

The best period $P_{\text {best }}=f_{\text {best }}^{-1}$ for each simulated data sample $\bar{t}^{*}$ was determined from the highest peak of the periodogram, $z\left(f_{\text {best }}\right)=\max [z(f)]$. In all our tests for the simulated data, only the $f_{\text {best }}$ values between $f_{\min }+f_{0} / 2$ and $f_{\max }-f_{0} / 2$ were accepted, which prevented the "detection" of possibly false $f_{\text {best }}$ values outside the tested range $\left[f_{\min }, f_{\max }\right]$.

\section{Rayleigh test for the simulated data}

The fully aperiodic simulations $(S=1)$ are discussed in Sect. 5.1. The fully periodic $(S=0)$ and the partly periodic $(S=1 / 3$ and $2 / 3)$ simulations are discussed in Sect. 5.2.

\subsection{Aperiodic cases $(S=1)$}

The statistics of the Rayleigh test were based on the "null hypothesis" $H_{0}$ formulated in Sect. 4. There would have been no reason to suspect these statistics if the detectability of the craters had remained constant in our data. Since, however, the detectability of older craters is lower, the statistics based on $H_{0}$ are not necessarily valid. To study this question, we performed simulations of fully aperiodic cases $(S=1$ in Eq. (9)), where the $A_{k}$ values were used to simulate this detectability effect. The Rayleigh test statistics based on the following four fully aperiodic hypotheses

$H_{1}$ : "the cumulative distribution function for the time points $t_{i}$ is $F_{\mathrm{AP}}\left(t, S=1, \sigma_{P}=0.05\right)$ ".

$\mathrm{H}_{2}$ : "the cumulative distribution function for the time points $t_{i}$ is $F_{\mathrm{AP}}\left(t, S=1, \sigma_{P}=0.1\right)$ ”.

$H_{3}$ : "the cumulative distribution function for the time points $t_{i}$ is $F_{\mathrm{AP}}\left(t, S=1, \sigma_{P}=0.2\right)$ ”. 
Table 3. Results of the fully aperiodic simulations for the preassigned significance levels $\gamma=0.01$ and 0.001 .

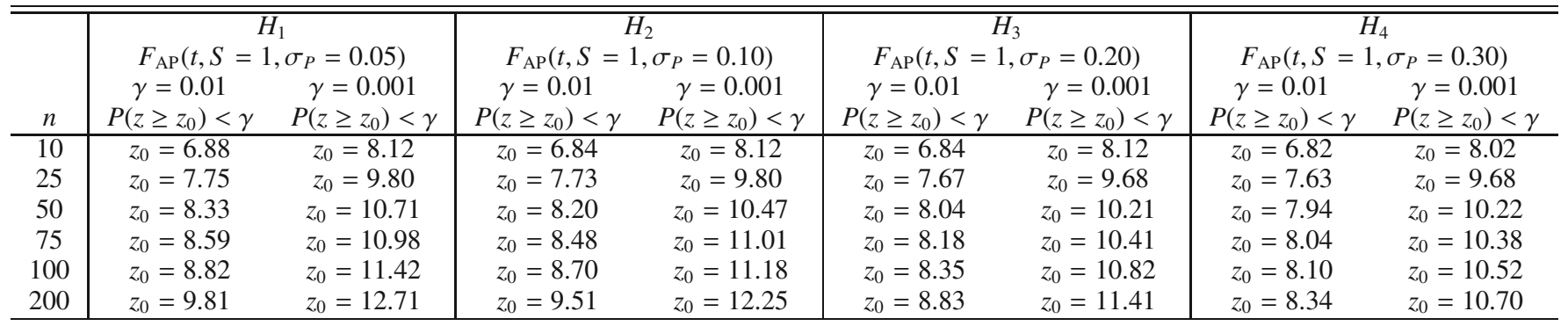

Table 4. The results of the fully periodic $(S=0)$ simulations.

\begin{tabular}{|c|c|c|c|c|c|c|c|c|}
\hline \multirow[b]{3}{*}{$n$} & "Real" & "False" & "Real' & "False" & "Real" & "False" & "Real" & "False" \\
\hline & \multicolumn{8}{|c|}{ Case 4A } \\
\hline & \multicolumn{2}{|c|}{$H_{1}$ rejected with $\gamma=0.01$} & $H_{0}$ rej & ith $\gamma=0$. & $H_{1}$ reje & th $\gamma=0.001$ & $H_{0}$ reje & th $\gamma=0.001$ \\
\hline 10 & 1.00 & 0.00 & 1.00 & 0.00 & 0.993 & 0.000 & 0.559 & 0.000 \\
\hline 25 & 1.00 & 0 & 1.00 & 0 & 1.000 & 0 & 1.000 & 0 \\
\hline 50 & 1.00 & 0 & 1.00 & 0 & 1.000 & 0 & 1.000 & 0 \\
\hline 75 & 1.00 & 0 & 1.00 & 0 & 1.000 & 0 & 1.000 & 0 \\
\hline 100 & 1.00 & 0 & 1.00 & 0 & 1.000 & 0 & 1.000 & 0 \\
\hline 200 & 1.00 & 0 & 1.00 & 0 & 1.000 & 0 & 1.000 & 0 \\
\hline
\end{tabular}

\begin{tabular}{r|cc|cccc|cc}
200 & $\mathbf{1 . 0 0}$ & 0 & $\mathbf{1 . 0 0}$ & 0 & $\mathbf{1 . 0 0 0}$ & 0 & $\mathbf{1 . 0 0 0}$ & 0 \\
\hline & \multicolumn{8}{c}{ Case 4B } \\
$n$ & \multicolumn{8}{c}{$H_{i}$ simulations with $F_{\mathrm{AP}}\left(t, S=0, \sigma_{P}=0.1\right)$} \\
\hline 10 & 0.70 & 0.01 & 0.67 & 0.01 & 0.267 & 0.002 & 0.030 & 0.000 \\
25 & $\mathbf{1 . 0 0}$ & 0.00 & $\mathbf{1 . 0 0}$ & 0.00 & $\mathbf{1 . 0 0 0}$ & 0.000 & $\mathbf{1 . 0 0 0}$ & 0.000 \\
50 & $\mathbf{1 . 0 0}$ & 0 & $\mathbf{1 . 0 0}$ & 0 & $\mathbf{1 . 0 0 0}$ & 0 & $\mathbf{1 . 0 0 0}$ & 0 \\
75 & $\mathbf{1 . 0 0}$ & 0 & $\mathbf{1 . 0 0}$ & 0 & $\mathbf{1 . 0 0 0}$ & 0 & $\mathbf{1 . 0 0 0}$ & 0 \\
100 & $\mathbf{1 . 0 0}$ & 0 & $\mathbf{1 . 0 0}$ & 0 & $\mathbf{1 . 0 0 0}$ & 0 & $\mathbf{1 . 0 0 0}$ & 0 \\
200 & $\mathbf{1 . 0 0}$ & 0 & $\mathbf{1 . 0 0}$ & 0 & $\mathbf{1 . 0 0 0}$ & 0 & $\mathbf{1 . 0 0 0}$ & 0 \\
\hline
\end{tabular}

\begin{tabular}{r|cc|cc|cc|cc}
\hline & \multicolumn{8}{c}{ Case $4 \mathrm{C}$} \\
$n$ & $H_{3}$ rejected with $\gamma=0.01$ & $H_{0}$ rejected with $\gamma=0.01$ & $H_{3}$ rejected with $\gamma=0.001$ & $H_{0}$ rejected with $\gamma=0.001$ \\
\hline 10 & 0.03 & 0.01 & 0.03 & 0.01 & 0.005 & 0.001 & 0.000 & 0.000 \\
25 & 0.31 & 0.02 & 0.44 & 0.03 & 0.119 & 0.003 & 0.173 & 0.006 \\
50 & 0.83 & $0.01 \downarrow$ & 0.92 & $0.01 \downarrow$ & 0.637 & 0.003 & 0.767 & 0.006 \\
75 & 0.98 & $0.00 \downarrow$ & $\mathbf{0 . 9 9}$ & $0.00 \downarrow$ & 0.935 & $0.002 \downarrow$ & 0.973 & $0.002 \downarrow$ \\
100 & $\mathbf{1 . 0 0}$ & $0.00 \downarrow$ & $\mathbf{1 . 0 0}$ & $0.00 \downarrow$ & 0.992 & $0.000 \downarrow$ & 0.998 & $0.000 \downarrow$ \\
200 & $\mathbf{1 . 0 0}$ & $0 \downarrow$ & $\mathbf{1 . 0 0}$ & $0 \downarrow$ & $\mathbf{1 . 0 0 0}$ & $0 \downarrow$ & $\mathbf{1 . 0 0 0}$ & $0 \downarrow$ \\
\hline
\end{tabular}

\begin{tabular}{r|cc|cc|cc|cc}
\hline & \multicolumn{8}{c}{ Case 4D } \\
$n$ & \multicolumn{8}{|c}{$t_{i}$ simulations with $F_{\mathrm{AP}}\left(t, S=0, \sigma_{P}=0.3\right)$} \\
\hline 10 & 0.00 & 0.01 & 0.00 & 0.01 & 0.000 & 0.001 & 0.000 & 0.000 \\
25 & 0.01 & 0.01 & 0.02 & 0.03 & 0.001 & 0.001 & 0.002 & 0.003 \\
50 & 0.02 & 0.02 & 0.06 & 0.05 & 0.005 & 0.002 & 0.013 & 0.007 \\
75 & 0.05 & 0.02 & 0.11 & 0.07 & 0.013 & 0.003 & 0.033 & 0.011 \\
100 & 0.09 & 0.03 & 0.19 & 0.08 & 0.027 & 0.004 & 0.066 & 0.015 \\
200 & 0.35 & 0.04 & 0.53 & 0.09 & 0.178 & 0.012 & 0.314 & 0.032 \\
\hline
\end{tabular}

\section{$H_{4}$ : "the cumulative distribution function for the time} points $t_{i}$ is $F_{\mathrm{AP}}\left(t, S=1, \sigma_{P}=0.3\right)$ ".

were simulated for the sample sizes $n=10,25,50,75,100$ and 200. Under each hypothesis $H_{1}, H_{2}, H_{3}$ or $H_{4}$, we performed the Rayleigh test for $Z=100000$ random samples of $\bar{t}^{*}=\left[t_{1}, \ldots, t_{n}\right]$ simulated from the corresponding fully aperiodic $F_{\mathrm{AP}}\left(t, S, \sigma_{P}\right)$. The periodogram $z(f)$ was calculated between $f_{\min }=0.5$ and $f_{\max }=2.0$ for each of these $\bar{t}^{*}$ samples. The highest peak $z_{0}=z\left(f_{\text {best }}\right)=\max [z(f)]$ of the periodogram for each simulated $\bar{t}^{*}$ sample, i.e. the global maximum of the periodogram, was then chosen. Finally, the ranking of all these $Z=100000$ values of $z_{0}$ determined the cumulative distribution function $P\left(z>z_{0}\right)=F(z)$, i.e. the probability that $z$ reached a value equal to, or higher than, $z_{0}$ under the hypothesis $H_{1}, H_{2}$, $\mathrm{H}_{3}$ or $\mathrm{H}_{4}$. The results of all these fully aperiodic simulations are compiled into Table 3 for the preassigned significance levels $\gamma=0.01$ and 0.001 .

\subsection{Periodic cases $(S=0,1 / 3$ and $2 / 3)$}

In this section, we determine how often the Rayleigh test detects the "real" $(P=1)$ or the "false" period $(P \neq 1)$ in the simulated crater data. The probability for the "reliable" detection of the "real" period is also investigated.

The cases $S=0$ (fully periodic), $1 / 3$ (partly periodic) and $2 / 3$ (partly periodic) were simulated for the sample sizes 
Table 5. Simulation results for the partly periodic cases $(S=1 / 3)$, otherwise as in Table 4 .

\begin{tabular}{|c|c|c|c|c|c|c|c|c|}
\hline & "Real" & "False" & "Real" & "False" & "Real" & "False" & "Real" & "False" \\
\hline & \multicolumn{8}{|c|}{$\begin{array}{c}\text { Case 5A } \\
\text { ns with } F_{\mathrm{AP}}(t, S=1\end{array}$} \\
\hline$n$ & \multicolumn{2}{|c|}{$H_{1}$ rejected with $\gamma=0.01$} & \multicolumn{2}{|c|}{$H_{0}$ rejected with $\gamma=0.01$} & \multicolumn{2}{|c|}{$H_{1}$ rejected with $\gamma=0.001$} & \multicolumn{2}{|c|}{$H_{0}$ rejected with $\gamma=0.001$} \\
\hline 10 & 0.20 & 0.01 & 0.21 & 0.01 & 0.104 & 0.002 & 0.032 & 0.000 \\
\hline 25 & 0.79 & 0.01 & 0.86 & 0.01 & 0.604 & 0.001 & 0.663 & 0.004 \\
\hline 50 & 0.99 & 0.00 & 1.00 & 0.00 & 0.978 & 0.000 & 0.992 & $0.001 \downarrow$ \\
\hline 75 & 1.00 & 0.00 & 1.00 & 0.00 & 1.000 & 0.000 & 1.000 & $0.000 \downarrow$ \\
\hline 100 & 1.00 & 0 & 1.00 & 0 & 1.000 & 0 & 1.000 & $0 \downarrow$ \\
\hline \multirow[t]{2}{*}{200} & 1.00 & 0 & 1.00 & 0 & 1.000 & 0 & 1.000 & $0 \downarrow$ \\
\hline & \multicolumn{8}{|c|}{$\begin{array}{l}\text { Case 5B } \\
\mathrm{h} F_{\mathrm{AP}}(t, S=\end{array}$} \\
\hline$n$ & \multicolumn{2}{|c|}{$\mathrm{H}_{2}$ rejected with $\gamma=0.01$} & \multicolumn{2}{|c|}{$H_{0}$ rejected with $\gamma=0.01$} & \multicolumn{2}{|c|}{$\mathrm{H}_{2}$ rejected with $\gamma=0.001$} & \multicolumn{2}{|c|}{$H_{0}$ rejected with $\gamma=0.001$} \\
\hline 10 & 0.10 & 0.01 & 0.10 & 0.01 & 0.027 & 0.002 & 0.003 & 0.000 \\
\hline 25 & 0.58 & 0.01 & 0.68 & 0.02 & 0.341 & 0.003 & 0.408 & 0.005 \\
\hline 50 & 0.97 & 0.00 & 0.99 & $0.00 \downarrow$ & 0.893 & $0.001 \downarrow$ & 0.950 & $0.002 \downarrow$ \\
\hline 75 & 1.00 & 0.00 & 1.00 & $0.00 \downarrow$ & 0.992 & $0.000 \downarrow$ & 0.998 & $0.000 \downarrow$ \\
\hline 100 & 1.00 & 0.00 & 1.00 & $0.00 \downarrow$ & 1.000 & $0.000 \downarrow$ & 1.000 & $0.000 \downarrow$ \\
\hline \multirow[t]{2}{*}{200} & 1.00 & 0 & 1.00 & $0 \downarrow$ & 1.000 & $0 \downarrow$ & 1.000 & $0 \downarrow$ \\
\hline & & & & \multicolumn{5}{|c|}{$t_{i}$ simulations with $F_{\mathrm{AP}}\left(t, S=1 / 3, \sigma_{P}=0.2\right)$} \\
\hline$\frac{n}{10}$ & \multicolumn{2}{|c|}{$H_{3}$ rejected with $\gamma=0.01$} & \multicolumn{2}{|c|}{$H_{0}$ rejected with $\gamma=0.01$} & \multicolumn{2}{|c|}{$H_{3}$ rejected with $\gamma=0.001$} & \multicolumn{2}{|c|}{$H_{0}$ rejected with $\gamma=0.001$} \\
\hline 25 & 0.06 & 0.02 & 0.09 & 0.03 & 0.013 & 0.002 & 0.019 & 0.004 \\
\hline 50 & 0.23 & 0.02 & 0.38 & 0.05 & 0.094 & 0.004 & 0.168 & 0.011 \\
\hline 75 & 0.48 & 0.02 & 0.65 & 0.05 & 0.266 & 0.006 & 0.411 & 0.014 \\
\hline 100 & 0.69 & 0.02 & 0.83 & $0.03 \downarrow$ & 0.456 & 0.006 & 0.645 & 0.014 \\
\hline 200 & 0.99 & $0.00 \downarrow$ & 1.00 & $0.00 \downarrow$ & 0.949 & $0.002 \downarrow$ & 0.986 & $0.003 \downarrow$ \\
\hline$n$ & \multicolumn{8}{|c|}{$\begin{array}{c}\text { Case 5D } \\
\text { ons with } F_{\mathrm{AP}}(t, S=\end{array}$} \\
\hline 10 & 0.00 & 0.01 & 0.00 & 0.02 & 0.000 & 0.001 & 0.000 & 0.000 \\
\hline 25 & 0.00 & 0.01 & 0.01 & 0.03 & 0.000 & 0.001 & 0.001 & 0.002 \\
\hline 50 & 0.01 & 0.01 & 0.02 & 0.04 & 0.001 & 0.001 & 0.003 & 0.005 \\
\hline 75 & 0.01 & 0.01 & 0.03 & 0.05 & 0.002 & 0.002 & 0.007 & 0.007 \\
\hline 100 & 0.02 & 0.02 & 0.05 & 0.06 & 0.003 & 0.002 & 0.012 & 0.009 \\
\hline 200 & 0.07 & 0.03 & 0.16 & 0.10 & 0.020 & 0.005 & 0.055 & 0.021 \\
\hline
\end{tabular}

$n=10,25,50,75,100$ and 200. For every fixed combination of $S, \sigma_{P}$, and $n$, we simulated $Z=100000$ random samples of $\bar{t}^{*}=\left[t_{1}, t_{2}, \ldots, t_{n}\right]$ from the respective cumulative distribution function $F_{\mathrm{AP}}\left(t, S, \sigma_{P}\right)$. The Rayleigh test periodogram $z(f)$ of each $\bar{t}^{*}$ sample was calculated between $f_{\min }=0.5$ and $f_{\text {max }}=2.0$. The detected best period $P_{\text {best }}=f_{\text {best }}^{-1}$ was identified from the one particular periodogram peak located at $f=f_{\text {best }}$ (i.e. the global maximum), which fulfilled $z\left(f_{\text {best }}\right)=\max [z(f)]$.

For each particular sample $\bar{t}^{*}$ simulated with some combination of $S, n$ and $\sigma_{P}$, the value of $z\left(f_{\text {best }}\right)$ was then compared to the numerical $z_{0}$ limit in the corresponding fully aperiodic $S=1$ case having the same $n$ and $\sigma_{P}$ values in Table 3 . If the criterion $z\left(f_{\text {best }}\right) \geq z_{0}$ was satisfied, the corresponding fully aperiodic hypothesis $H_{1}, H_{2}, H_{3}$ or $H_{4}$ was rejected.

The results of all simulations for the fully periodic $(S=$ 0 ) case are given in Table 4 . The respective results for the partly periodic cases $(S=1 / 3$ and 2/3) are given in Tables 5 and 6. All notations in Tables 4-6 are the same. Column $1(n)$ is the number of simulated time points $\bar{t}^{*}=\left[t_{1}, t_{2}, \ldots, t_{n}\right]$ generated with corresponding cumulative distribution function $F_{\mathrm{AP}}\left(t, S, \sigma_{P}\right)$. Column 2 ("real") is the probability for rejecting one of the hypothesis $H_{1}, H_{2}, H_{3}$ or $H_{4}$ with $\gamma=0.01$ for the "real" period $P=1$. Detections marked with 0.99 or 1.00 (in bold face) are "reliable" as explained later. Column 3 ("false") is the probability for rejecting one of the hypothesis $H_{1}, H_{2}, H_{3}$ or $H_{4}$ with $\gamma=0.01$ for the "false" periods $P \neq 1$. An integer value " 0 " indicates that the corresponding hypothesis was not rejected even once in all simulations. Columns 4 ("real") and 5 ("false") give the probability for rejecting $H_{0}$ with $\gamma=0.01$, otherwise as in Cols. 2 and 3. Columns 6 ("real"), 7 ("false"), 8 ("real") and 9 ("false") were the results with $\gamma=0.001$, otherwise as in Cols. 2-5. The symbol " $\downarrow$ " denotes the cases where the probability of detecting "false" periodicities first increased and then decreased as a function of the sample size $n$, i.e. the cases where the "real" period was emerging, as explained in Sect. 6.

For clarity, the cases of all $S$ and $\sigma_{P}$ combinations in these periodic simulations have been named in the corresponding tables. These names provide an easy way to refer to any particular simulated case. For example, the $z_{0}$ values of $H_{1}$ (Table 3: $\left.S=1, \sigma_{P}=0.05\right)$ were compared to the $z\left(f_{\text {best }}\right)$ values of Case 4A (Table 4: $S=0, \sigma_{P}=0.05$ ), Case 5A (Table 5: $S=$ $1 / 3, \sigma_{P}=0.05$ ) and Case 6A (Table 6: $S=2 / 3, \sigma_{P}=0.05$ ).

The analytical estimate for the critical level $Q$ for all $z\left(f_{\text {best }}\right)$ values were also calculated from Eq. (11). The "null hypothesis" $H_{0}$ was rejected, if and only if $Q<\gamma$ (Eq. (12)).

When $H_{0}, H_{1}, H_{2}, H_{3}$, or $H_{4}$ was rejected with $\gamma=0.01$ or 0.001 for some particular simulated sample $\bar{t}^{*}$, we also checked if the inverse $P_{\text {best }}=f_{\text {best }}^{-1}$ was equal to the period $P=1$ 
Table 6. Simulation results for the partly periodic cases $(S=2 / 3)$, otherwise as in Table 4 .

\begin{tabular}{|c|c|c|c|c|c|c|c|c|}
\hline \multirow[b]{3}{*}{$n$} & "Real" & "False" & "Real" & "False" & "Real" & "False" & "Real" & "False" \\
\hline & \multicolumn{8}{|c|}{$\begin{array}{c}\text { Case 6A } \\
\text { as with } F_{\mathrm{AP}}(t, S=2\end{array}$} \\
\hline & \multicolumn{2}{|c|}{$H_{1}$ rejected with $\gamma=0.01$} & \multicolumn{2}{|c|}{$H_{0}$ rejected with $\gamma=0.0$} & \multicolumn{2}{|c|}{$H_{1}$ rejected with $\gamma=0.001$} & \multicolumn{2}{|c|}{$H_{0}$ rejected with $\gamma=0.001$} \\
\hline 10 & 0.02 & 0.01 & 0.02 & 0.01 & 0.004 & 0.001 & 0.001 & 0.000 \\
\hline 25 & 0.08 & 0.01 & 0.12 & 0.04 & 0.024 & 0.002 & 0.034 & 0.004 \\
\hline 50 & 0.26 & 0.02 & 0.42 & 0.05 & 0.114 & 0.003 & 0.219 & 0.012 \\
\hline 75 & 0.50 & 0.02 & 0.68 & 0.05 & 0.289 & 0.004 & 0.470 & 0.015 \\
\hline 100 & 0.69 & $0.01 \downarrow$ & 0.84 & $0.03 \downarrow$ & 0.472 & 0.005 & 0.688 & 0.015 \\
\hline \multirow[t]{2}{*}{200} & 0.98 & $0.00 \downarrow$ & 1.00 & $0.00 \downarrow$ & 0.930 & $0.001 \downarrow$ & 0.987 & $0.003 \downarrow$ \\
\hline & \multicolumn{8}{|c|}{ Case 6B } \\
\hline$n$ & \multicolumn{2}{|c|}{$\mathrm{H}_{2}$ rejected with $\gamma=0.01$} & \multicolumn{2}{|c|}{$H_{0}$ rejected with $\gamma=0.01$} & \multicolumn{2}{|c|}{$\mathrm{H}_{2}$ rejected with $\gamma=0.001$} & \multicolumn{2}{|c|}{$H_{0}$ rejected with $\gamma=0.001$} \\
\hline 10 & 0.01 & 0.01 & 0.01 & 0.01 & 0.001 & 0.001 & 0.000 & 0.000 \\
\hline 25 & 0.04 & 0.01 & 0.07 & 0.04 & 0.010 & 0.002 & 0.016 & 0.003 \\
\hline 50 & 0.15 & 0.02 & 0.27 & 0.06 & 0.053 & 0.003 & 0.112 & 0.011 \\
\hline 75 & 0.31 & 0.02 & 0.50 & 0.06 & 0.137 & 0.004 & 0.279 & 0.016 \\
\hline 100 & 0.48 & 0.02 & 0.69 & 0.05 & 0.272 & 0.005 & 0.471 & 0.017 \\
\hline \multirow[t]{2}{*}{200} & 0.91 & $0.01 \downarrow$ & 0.97 & $0.01 \downarrow$ & 0.779 & $0.004 \downarrow$ & 0.930 & $0.009 \downarrow$ \\
\hline & \multicolumn{8}{|c|}{ Case 6C } \\
\hline$n$ & \multicolumn{2}{|c|}{$H_{3}$ rejected with $\gamma=0.01$} & \multicolumn{2}{|c|}{$H_{0}$ rejected with $\gamma=0.01$} & \multicolumn{2}{|c|}{$H_{3}$ rejected with $\gamma=0.001$} & \multicolumn{2}{|c|}{$H_{0}$ rejected with $\gamma=0.001$} \\
\hline 10 & 0.01 & 0.01 & 0.01 & 0.01 & 0.000 & 0.001 & 0.000 & 0.000 \\
\hline 25 & 0.01 & 0.01 & 0.01 & 0.03 & 0.001 & 0.001 & 0.002 & 0.003 \\
\hline 50 & 0.01 & 0.01 & 0.04 & 0.05 & 0.003 & 0.002 & 0.008 & 0.006 \\
\hline 75 & 0.03 & 0.02 & 0.08 & 0.06 & 0.007 & 0.002 & 0.021 & 0.010 \\
\hline 100 & 0.05 & 0.02 & 0.13 & 0.07 & 0.013 & 0.003 & 0.041 & 0.013 \\
\hline \multirow[t]{2}{*}{200} & 0.20 & 0.03 & 0.39 & 0.10 & 0.074 & 0.006 & 0.199 & 0.028 \\
\hline & \multicolumn{8}{|c|}{$\begin{array}{l}\text { Case 6D } \\
\mathrm{h} F_{\mathrm{AP}}(t, S=\end{array}$} \\
\hline 10 & 0.00 & 0.01 & 0.00 & 0.01 & 0.000 & 0.001 & 0.000 & 0.000 \\
\hline 25 & 0.00 & 0.01 & 0.00 & 0.03 & 0.000 & 0.001 & 0.000 & 0.002 \\
\hline 50 & 0.00 & 0.01 & 0.01 & 0.04 & 0.000 & 0.001 & 0.001 & 0.004 \\
\hline 75 & 0.00 & 0.01 & 0.01 & 0.04 & 0.000 & 0.001 & 0.001 & 0.006 \\
\hline 100 & 0.00 & 0.01 & 0.01 & 0.05 & 0.000 & 0.001 & 0.002 & 0.006 \\
\hline 200 & 0.01 & 0.01 & 0.02 & 0.07 & 0.001 & 0.002 & 0.004 & 0.010 \\
\hline
\end{tabular}

used in our simulations. The criterion for accepting the detected period $P_{\text {best }}$ as the "real" one was

$1-\frac{f_{0}}{2} \leq f_{\text {best }} \leq 1+\frac{f_{0}}{2}$

Hence the criterion for "false" detections was

$f_{\text {best }}<1-\frac{f_{0}}{2}$ or $1+\frac{f_{0}}{2}<f_{\text {best }}$.

Finally, the number of simulated samples, where the "real" period was detected, was divided by the number of all simulated samples $Z=100000$. The detection of the "real" period was accepted as "reliable", if the following criterion was satisfied:

The hypothesis $\mathrm{H}_{0}, \mathrm{H}_{1}, \mathrm{H}_{2}, \mathrm{H}_{3}$, or $\mathrm{H}_{4}$ was rejected with the "real" periodicity at the preassigned significance level $\gamma$ in at least $(1-\gamma) Z$ of all $Z$ simulated $\bar{t}^{*}$ samples.

One particular example of all our simulations is given in Fig. 1, where the cumulative distribution function $F_{\mathrm{AP}}\left(t, S, \sigma_{P}\right)$ is displayed for $S=1 / 3$ and $\sigma_{P}=0.1$. This cumulative distribution function was used to simulate one arbitrary random sample $\bar{t}^{*}=\left[t_{1}, t_{2}, \ldots, t_{n}\right](n=25)$. The periodogram $z(f)$ for this random sample $\bar{t}^{*}$ is shown in Fig. 2. The highest peak of the periodogram $z(f)$ is within the frequency range $\left[1-f_{0} / 2,1+f_{0} / 2\right]$, i.e. the best period $P_{\text {best }}$ detected from these particular simulated data is "real". When the critical level $Q$ of this peak is calculated from the Eq. (11), the hypothesis $H_{0}$ has to be rejected with $\gamma=0.01$ (i.e. the peak exceeds the lower continuous horizontal line), but not with $\gamma=0.001$ (i.e. the peak does not exceed the higher continuous horizontal line). The corresponding simulated fully aperiodic hypothesis is $\mathrm{H}_{2}$, where the cumulative distribution was $F_{\mathrm{AP}}\left(t, S=1, \sigma_{P}=0.1\right)$. Under $H_{2}$, the probabilities that $z\left(f_{\text {best }}\right)$ reaches $z_{0}=7.73$ or 9.80 are $\gamma=0.01$ or 0.001 (Table 3: Cols. 4 and $5, n=25$ ). This highest peak of the periodogram at $f_{\text {best }}$ exceeds only the former level (i.e. lower dotted horizontal line), but not the latter (i.e. higher dotted horizontal line). Hence $\mathrm{H}_{2}$ is rejected with $\gamma=0.01$, but not with $\gamma=0.001$. Finally, the results for all $Z=100000$ data samples $\bar{t}^{*}=\left[t_{1}, t_{2}, \ldots, t_{n}\right](n=25)$ simulated with $F_{\mathrm{AP}}\left(t, S=1 / 3, \sigma_{P}=0.10\right)$ are given in Table 5 (Case 5B: $n=25)$. The probability for detecting the "real" periodicity with $\gamma=0.01$ is 0.58 (under $H_{2}$ ) and 0.68 (under $H_{0}$ ), while the respective probabilities for detecting "false" periodicities are 0.01 (under $\mathrm{H}_{2}$ ) and 0.02 (under $H_{0}$ ). With $\gamma=0.001$, the probability for detecting the "real" periodicity is 0.341 (under $H_{2}$ ) and 0.408 (under $H_{0}$ ). Table 5 also reveals that the detection of the "real" periodicity $P=1$ would be "reliable", if the sample size reached $n \geq 75$ or $n \geq 50$ (Case 5B: $H_{2}$ or $H_{0}$ rejected with $\gamma=0.01$ : marked 1.00 or 0.99 ) and $n \geq 100$ (Case 5B: $H_{2}$ or $H_{0}$ rejected with $\gamma=0.001$ : both marked 1.000), respectively.

Jetsu (1997) showed that the rounding of the impact crater data induces a "human-signal", i.e. the detection of spurious 


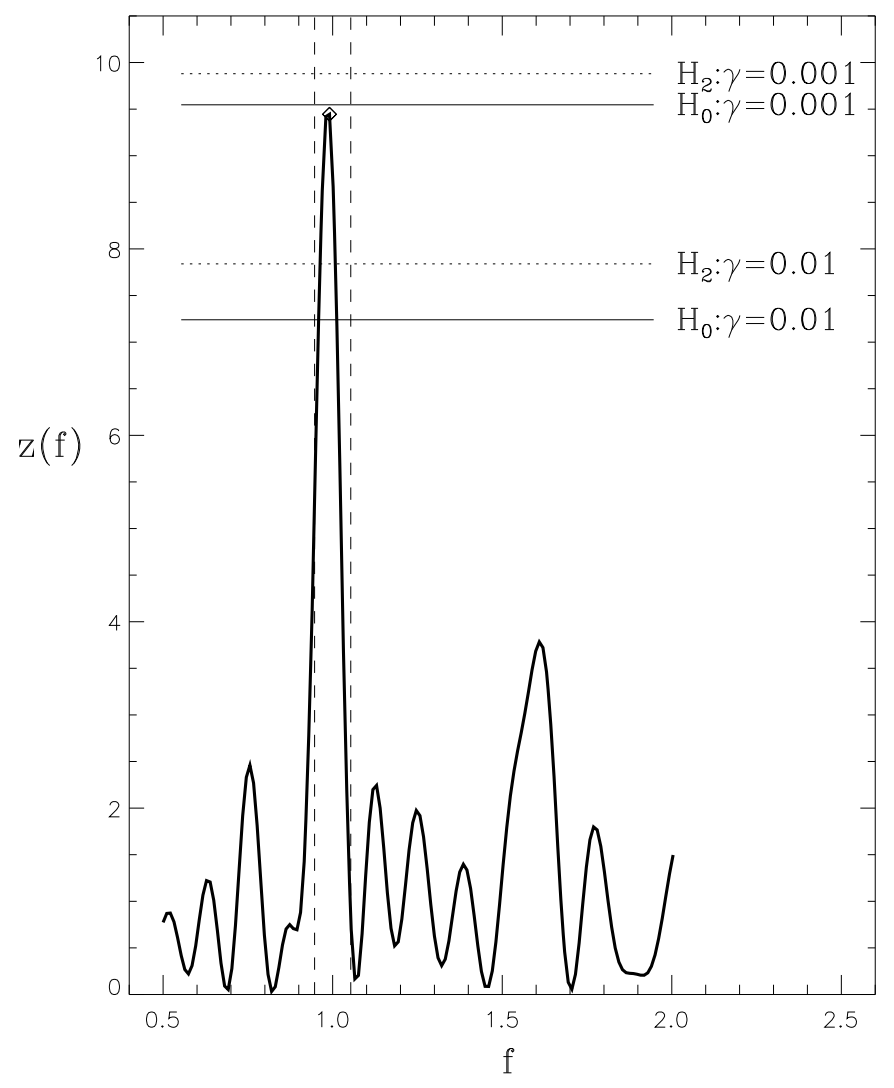

Fig. 2. One arbitrary random sample $\bar{t}^{*}=\left[t_{1}, t_{2}, \ldots, t_{n}\right](n=25)$ was simulated from the cumulative distribution function $F_{\mathrm{AP}}\left(t, S, \sigma_{P}\right)$ of Fig. 1. The thick continuous line is the Rayleigh test periodogram $z(f)$ of these data between $f_{\min }=0.5$ and $f_{\max }=2.0$. The global maximum $z\left(f_{\text {best }}\right)$ (denoted with a diamond) is located between $1-f_{0} / 2$ and $1+f_{0} / 2$ (denoted with two vertical dashed lines), i.e. the best period $P_{\text {best }}=f_{\text {best }}^{-1}$ fulfills the criterion of the "real" periodicity. This maximum $z\left(f_{\text {best }}\right)$ reaches the $\gamma=0.01$ levels for rejecting $H_{0}$ (lower continuous horizontal line) or $H_{2}$ (lower dotted horizontal line $\equiv z_{0}=7.73$ in Table 3). However, the $\gamma=0.001$ levels for rejecting $H_{0}$ (higher continuous horizontal line) or $H_{2}$ (higher dotted horizontal line $\equiv z_{0}=9.80$ in Table 3) are not reached.

periodicities like 3, 5, 10 or 20 Myr. The effects of this rounding to the statistics and to the significance estimates of the detected periodicities were then studied in Jetsu \& Pelt (2000). We decided to round the simulated $t_{i}$ values into integer multiples of $(3 / \pi) \sigma_{P} \approx 0.95 \sigma_{P}$. The factor $3 / \pi$ was applied, because this prevented that some simulated rounded $t_{i}$ would have been integers, i.e. multiples of the "real" periodicity $P=1$. The results of these simulations for rounded data were compiled into Tables 7-9. For example, the simulations of Case 4A in Table 4 were otherwise same as those of Case 7A in Table 7, except that in the latter case the $t_{i}$ were rounded into integer multiples of $(3 / \pi) \sigma_{P}$, where $\sigma_{P}=0.05$.

\section{Results and discussion}

The simulated periodicity was $P=f^{-1}=1$. The Rayleigh tests were applied between $f_{\min }=0.5$ and $f_{\max }=2.0$. This combination was ideal. Let us assume that the tested frequency range had remained the same, but the simulated periodicity would have been $P=1.8 \equiv f=0.56$. In this case, the $z(f)$ periodogram would peak at $f=0.56=1.8^{-1}, f=1.11=0.9^{-1}$ and $f=1.66=0.6^{-1}$, because these frequencies give similar phase distributions. In the fully periodic case, it might have been possible to notice the effect that these fractions of the correct periodicity concentrated the $t_{i}$ on every other $(P=0.9)$ or every third $(P=0.6)$ round. But this effect would have gone unnoticed, if there also was an aperiodic component in the data. With this $P=1.8$ periodicity, the detection of the fractions of the "real" periodicity would certainly have increased the probability of detecting "false" periodicities. Hence the location of "real" periodicity within the tested range influences the probability for "real" or "false" detections. Furthermore, if the tested range had been longer, there would have been more problems with these fractions, as well as problems connected to the rounding of the data. For example, the rounding of $t_{i}$ with $\sigma_{P}=0.3$ would have introduced a spurious frequency $f \approx 3.33$, which was "conveniently" outside our tested range. For obvious reasons, no "reliable" detections of "false" periods occurred in Tables 4-9. But the above rounding with $\sigma_{P}=0.3$ would have caused a "reliable" detection of the "false" spurious frequency $f \approx 3.33$, because the phases $\phi_{i}$ calculated with this $f$ would have been equal for all rounded $t_{i}$.

Even though we studied only some limited combinations of $K, n, \sigma_{P}, S, P, P_{\min }$ and $P_{\max }$, we have in fact formulated a method to determine the probability of "reliable" detection of any periodicity in any type of impact crater data. This method also includes effects of erosion and the presence of an aperiodic component. It reveals, if the fractions of "real" periodicity influence the probability of "reliable" detection, or enhance detection "false" periodicities. Let us assume that the tested data are $t_{1} \pm$ $\sigma_{1}, t_{2} \pm \sigma_{2}, \ldots, t_{\mathrm{n}} \pm \sigma_{\mathrm{n}}$, while the period $P_{K}$ has been detected in a Rayleigh test between $f_{\min }=P_{\max }^{-1}$ and $f_{\max }=P_{\min }^{-1}$. A suitable estimate for the number of cycles is $K=\operatorname{INT}\left[\left(t_{n}-t_{1}\right) / P_{K}\right]$. The $A_{k}$ values can now be calculated as in Table 2. The error in the simulations is fixed to $\sigma_{P}=\sigma_{P^{\prime}}$ of Eq. (1). The simulations are first performed with $S=1$, i.e. for the fully aperiodic case. A large number (e.g. $Z=100000$ ) of samples of $n$ simulated time point samples $\bar{t}^{*}=\left[t_{1}, t_{2}, \ldots, t_{n}\right]$ are generated from $F_{\mathrm{AP}}\left(t, S=1, \sigma_{P}=\sigma_{P^{\prime}}\right)$ of Eq. (9). Since the simulated periodicity is $P=1$, the Rayleigh test range must be fixed to $f_{\min }^{\prime}=\left(P_{\max } / P_{K}\right)^{-1}+f_{0} / 2$ and $f_{\max }^{\prime}=\left(P_{\min } / P_{K}\right)^{-1}-f_{0} / 2$, where $f_{0}=P_{K} /\left(t_{n}-t_{1}\right)$ is the distance between independent frequencies. The highest peak $z_{0}=\max [z(f)]$ of the Rayleigh test is identified for each $\bar{t}^{*}$ sample. All these $Z$ values of $z_{0}$ are used to determine the limits for $z(f)$ reaching the chosen preassigned significance level $\gamma$, like in Table 3. Finally, the probability of "reliable" detection for any value of $S<1$ can be obtained with the same approach as already outlined in Sect. 5.2 (see also Fig. 2).The "real" periodicity in all these simulations is $P=1$. Because the contribution of the aperiodic component is unknown, it is logical to begin these simulations from $S=0$. This will reveal the possibility for "reliable" detection, if all data were fully periodic.

If all impacts in Table 1 were periodic, "reliable" periodicity detection would be possible in $C_{2}$ with $\gamma=0.01$ and 0.001 (comparable to $n=25$ of Case 4B in Table 4), and impossible in $C_{3}$ (comparable to $n=50$ of Case $4 \mathrm{C}$ in Table 4). But "reliable" detections would be impossible, if one or two thirds of these impacts were aperiodic (see Tables 5 and 6).

As the first example, we performed the Rayleigh test for the subsample $C_{2}$ of data in Table 1, because "reliable" detection could be possible in the fully periodic case. The tested period range was between 15 and $60 \mathrm{Myr}$, because this combination was comparable to the frequency range $[0.5,2.0]$ used in our simulations, if the "real" periods were close to $30 \mathrm{Myr}$. The best period $P_{K}=34.2$ reached the critical level $Q=0.29$ (Eq. (11)), i.e. $H_{0}$ was not rejected. Nor was $H_{2}$. The maximum value for the test 
Table 7. Simulation results for the fully periodic cases $(S=0)$ for the rounded data, otherwise as in Table 4 .

\begin{tabular}{|c|c|c|c|c|c|c|c|c|}
\hline & "Real" & "False" & "Real" & "False" & "Real" & "False" & "Real" & "False" \\
\hline & \multicolumn{8}{|c|}{$\begin{array}{c}\text { Case 7A } \\
\left.t, S=0, \sigma_{P}=0.05\right) \mathrm{rou}\end{array}$} \\
\hline$n$ & \multicolumn{2}{|c|}{$H_{1}$ rejected with $\gamma=0.01$} & \multicolumn{2}{|c|}{$H_{0}$ rejected with $\gamma=0.01$} & \multicolumn{2}{|c|}{$H_{1}$ rejected with $\gamma=0.001$} & \multicolumn{2}{|c|}{$H_{0}$ rejected with $\gamma=0.001$} \\
\hline 10 & 1.00 & 0.00 & 1.00 & 0.00 & 0.993 & 0.000 & 0.510 & 0.000 \\
\hline 25 & 1.00 & 0 & 1.00 & 0 & 1.000 & 0 & 1.000 & 0 \\
\hline 50 & 1.00 & 0 & 1.00 & 0 & 1.000 & 0 & 1.000 & 0 \\
\hline 75 & 1.00 & 0 & 1.00 & 0 & 1.000 & 0 & 1.000 & 0 \\
\hline 100 & 1.00 & 0 & 1.00 & 0 & 1.000 & 0 & 1.000 & 0 \\
\hline 200 & 1.00 & 0 & 1.00 & 0 & 1.000 & 0 & 1.000 & 0 \\
\hline$n$ & \multicolumn{8}{|c|}{$\begin{array}{c}\text { Case 7B } \\
\left.0, \sigma_{P}=0.1\right) \mathrm{ro}\end{array}$} \\
\hline 10 & 0.64 & 0.01 & 0.62 & 0.01 & 0.228 & 0.002 & 0.024 & 0.000 \\
\hline 25 & 1.00 & 0.00 & 1.00 & 0.00 & 1.000 & 0.000 & 1.000 & 0.000 \\
\hline 50 & 1.00 & 0 & 1.00 & 0 & 1.000 & 0 & 1.000 & 0 \\
\hline 75 & 1.00 & 0 & 1.00 & 0 & 1.000 & 0 & 1.000 & 0 \\
\hline 100 & 1.00 & 0 & 1.00 & 0 & 1.000 & 0 & 1.000 & 0 \\
\hline 200 & 1.00 & 0 & 1.00 & 0 & 1.000 & 0 & 1.000 & 0 \\
\hline$n$ & $\mathrm{H}_{3}$ reje & $\begin{array}{r}t_{i} \operatorname{sim} \mathrm{I} \\
\text { ith } \gamma=0.01\end{array}$ & $\begin{array}{r}\text { ations w } \\
H_{0} \text { rej }\end{array}$ & $\begin{array}{l}(t, S=0, \sigma \\
\text { ith } \gamma=0.0\end{array}$ & $\begin{array}{l}\mathrm{e} 7 \mathrm{C} \\
=0.2) \text { rol } \\
H_{3} \text { reje }\end{array}$ & $\begin{array}{l}\text { o multiple } \\
\text { th } \gamma=0.0\end{array}$ & $\begin{array}{l}(3 / \pi) \sigma_{P} \\
H_{0} \text { rejec }\end{array}$ & th $\gamma=0.001$ \\
\hline 10 & 0.03 & 0.01 & 0.03 & 0.01 & 0.003 & 0.001 & 0.000 & 0.000 \\
\hline 25 & 0.24 & 0.02 & 0.33 & 0.03 & 0.085 & 0.003 & 0.123 & 0.005 \\
\hline 50 & 0.74 & $0.01 \downarrow$ & 0.86 & $0.02 \downarrow$ & 0.511 & 0.004 & 0.656 & 0.008 \\
\hline 75 & 0.95 & $0.00 \downarrow$ & 0.98 & $0.01 \downarrow$ & 0.865 & 0.003 & 0.934 & $0.004 \downarrow$ \\
\hline 100 & 0.99 & $0.00 \downarrow$ & 1.00 & $0.00 \downarrow$ & 0.974 & $0.001 \downarrow$ & 0.992 & $0.001 \downarrow$ \\
\hline 200 & 1.00 & $0 \downarrow$ & 1.00 & $0 \downarrow$ & 1.000 & $0 \downarrow$ & 1.000 & $0 \downarrow$ \\
\hline$n$ & \multicolumn{8}{|c|}{$\begin{array}{c}\text { Case 7D } \\
\left.t, S=0, \sigma_{P}=0.3\right) \mathrm{ro}\end{array}$} \\
\hline$\overline{10}$ & 0.00 & 0.01 & 0.00 & 0.01 & 0.000 & 0.003 & 0.000 & 0.002 \\
\hline 25 & 0.01 & 0.01 & 0.01 & 0.03 & 0.001 & 0.002 & 0.002 & 0.004 \\
\hline 50 & 0.01 & 0.02 & 0.04 & 0.05 & 0.003 & 0.002 & 0.007 & 0.008 \\
\hline 75 & 0.03 & 0.02 & 0.07 & 0.07 & 0.007 & 0.004 & 0.018 & 0.011 \\
\hline 100 & 0.05 & 0.02 & 0.12 & 0.08 & 0.014 & 0.004 & 0.037 & 0.016 \\
\hline 200 & 0.21 & 0.04 & 0.37 & 0.11 & 0.087 & 0.010 & 0.180 & 0.031 \\
\hline
\end{tabular}

statistic was $z_{0}=3.56$, which was much lower than the closest comparable value in Table $3\left(H_{2}\right.$ rejection with $\gamma=0.01$ would have required $z_{0}=7.73$ for $\sigma_{P}=0.10$ and $n=25$ ).

As the second example, we studied the data in Alvarez \& Muller (1984), which consisted of eleven impact craters. Because the ages of Logoisk and Boltysh craters were equal in the data given in their article, the actual sample size was $n=10$. Their detected period $P_{K}=28.4 \mathrm{Myr}$ and the errors for the crater ages gave $\sigma_{P^{\prime}}=0.240 \approx 0.2$ (Eq. (1)). In the fully periodic case, the probability of "reliable" detection with $\gamma=0.01$ was only 0.03 (Table 4: Case 4C, $n=10$ ). In the partly aperiodic cases, the respective probability was 0.01 for both cases (Table 5: Case 5C and Table 6: Case 6C, $n=10$ ). Hence the detection of the 28.4 Myr periodicity was far from "reliable". The above probabilities were in fact even lower, because Alvarez \& Muller (1984) tested the range between 10 and $100 \mathrm{Myr}$, i.e. their range was $10 / 28.4=0.4$ and $100 / 28.4=3.5$ in the units of $P_{K}$, which was a much longer range than in our simulations. Jetsu \& Pelt (2000) performed a Rayleigh test between 10 and $100 \mathrm{Myr}$ for the same data and detected the 28.54 Myr period, which reached the critical level of $Q=0.034$.

As the third example, a subsample

$S_{1}: t_{\mathrm{i}} \leq 250, \sigma_{i} \leq 10, D_{i} \geq 35$.

was selected from the data in Table 1. Stothers (2006) detected a 35 Myr periodicity from such a subsample. They applied this criterion, because Shoemaker et al. (1990) had argued that larger craters were more probably caused by comet impacts. The $S_{1}$ criterion actually gave a further subsample of the subsample selected with our $C_{2}$ criterion. We performed the Rayleigh test to the $n=13$ craters selected with $S_{1}$ from Table 1 . The tested range was between 22 and $50 \mathrm{Myr}$, as in Stothers (2006). The best period $P_{K}=34.94 \mathrm{Myr}$ reached the critical level $Q=0.22$ (Eq. (11)). Hence there was no significant periodicity in these data, besides what could be described as "noise". The test statistic maximum was only $z_{0}=3.02$. One could argue that the statistics based on $H_{0}$ were not reliable for such a small sample $(n=$ 13). But even this argument would fail, because the rejection of $H_{1}$ with $\gamma=0.01$ would have required values close to $z_{0}=6.88$ (see Table 3: $\sigma_{P}=0.05, n=10$ ). Furthermore, the tested range was short, between $22 / 34.94=0.63$ and 50/34.94 $=1.43$ in units of $P_{K}$. Therefore "reliable" detection with $\gamma=0.01$ would have succeeded in the fully periodic case $(S=0)$, because these data were very accurate having $\sigma_{P^{\prime}}=0.064$ with $P_{K}=34.94$ (comparable to Case 4A, $n=10$ in Table 4). In conclusion, had significant periodicity been present, we would certainly have detected it.

A sequence of random variables is called heteroscedastic, if the variances of these variables are different. This was more or less obvious in our study, because the accuracy decreased for older craters. It might have been possible to simulate this effect by using a different $\sigma_{P}$ value during each cycle $k=1, \ldots, K$. 
Table 8. Simulation results for the partly periodic cases $(S=1 / 3)$ for the rounded data, otherwise as in Table 4.

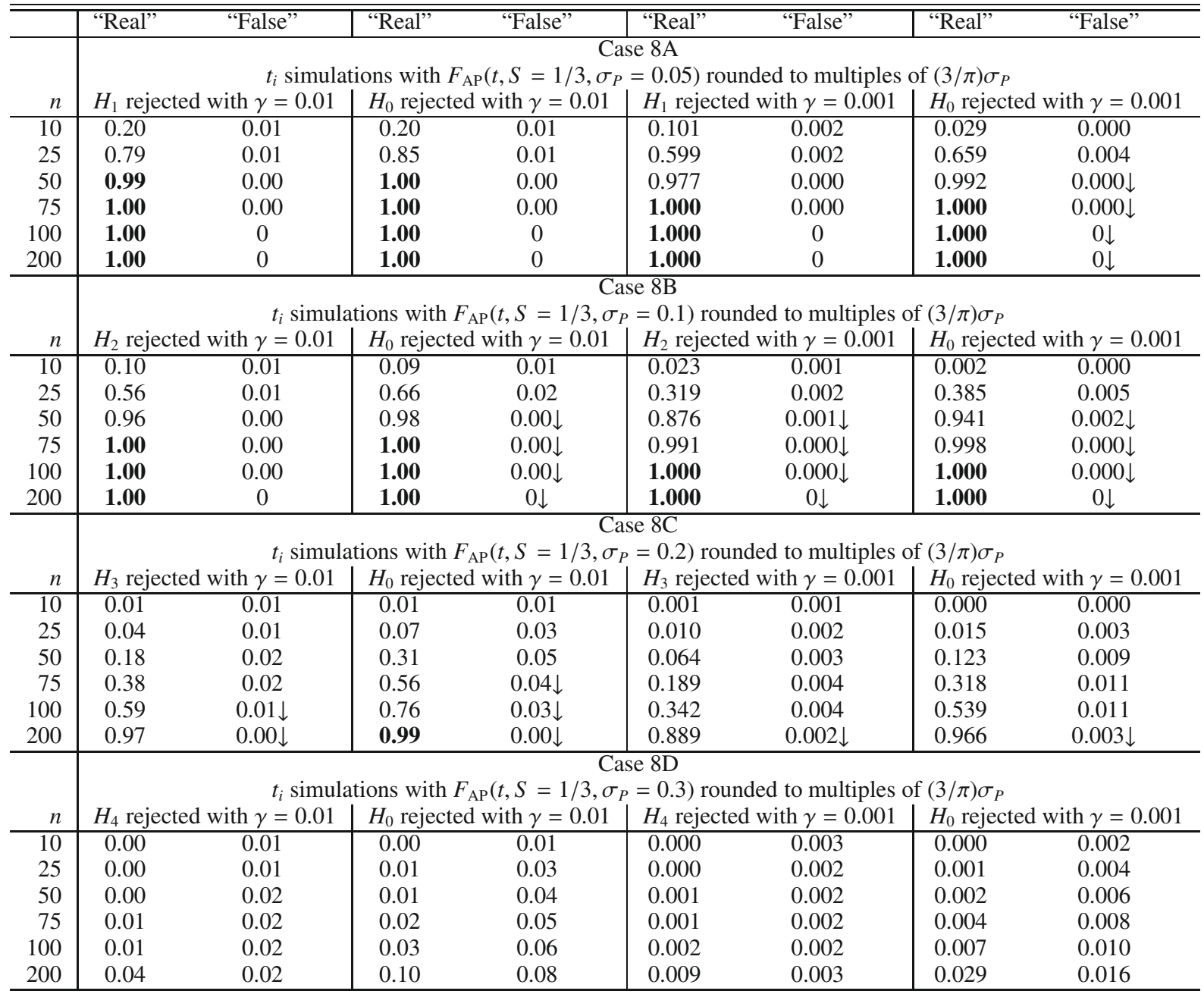

The distributions of Eqs. (2) and (3) with these particular $\sigma_{P}$ values could then have been convolved separately to the aperiodic and periodic components within each cycle. But in this case there would have been no unique simulated $\sigma_{P}$ value that could have been compared with the unique $\sigma_{P^{\prime}}$ value calculated from the data (Eq. (1)). Deutsch \& Schaerer (1994) noted that the error estimates in the crater data at the time of the initial discovery of the alleged periodicity might have been too small. This view is supported by the comparison of Tables 1 and 10. The more accurate crater ages derived using modern Ar-Ar dating techniques, described e.g. by Kelley (2003a) and Kelley (2003b), clearly indicate that some errors in the earlier ages exceeded the $\pm 3 \sigma_{i}$ estimate (e.g. Shunak: from $12 \pm 5$ to $45 \pm 10 \mathrm{Myr}$, Boltysh: from $88 \pm 3$ to $65.17 \pm 0.64 \mathrm{Myr}$, or Zapdanaya: from $115 \pm$ 7.1 to $165 \pm 5 \mathrm{Myr}$ ). Although the accuracy of the dating has improved over the years, these large and heteroscedastic errors still prevent "reliable" detection of periodicity.

Our simulations clearly revealed that the statistics based on $H_{0}$ were less reliable than those based on $H_{1}, H_{2}, H_{3}$ or $H_{4}$. This can be seen from the following points:

Firstly, Case 6D in Table 6 was dominated by the aperiodic component $(S=2 / 3)$ and the error was large $\left(\sigma_{P}=0.3\right)$, i.e. this case was very close to being fully aperiodic. Under $H_{4}$, the sums for the probabilities for detecting the "false" or "real" periodicity were close to $\gamma=0.01$ or 0.001 . Under $H_{0}$, these sums clearly exceeded $\gamma$.
Secondly, the rejection of $H_{0}$ was always more probable than that of $H_{1}, H_{2}, H_{3}$ or $H_{4}$, except for $n=10$. This effect occurred for both $\gamma=0.01$ and 0.001 in all Tables 4-9. For example, Brazier (1994) have noted that with small samples $(n<50)$ the assumption of asymptotic probability density distribution $f(z)=$ $\mathrm{e}^{-z}$ of the Rayleigh power $z$ does not hold. This explained the above exception with $n=10$.

Thirdly, when erosion was introduced into the simulations, the statistics differed from those based on $H_{0}$. The effect of erosion was simulated with the $A_{k}$ estimates. We chose to give one example which should clarify how erosion can alter the statistics. If $A_{1}$ were much larger than $A_{2}, A_{3}, \ldots A_{10}$, then values of $0<t_{i}<1$ would be more probable than in the case $A_{1}=A_{2}=\ldots=A_{10}=0.1$, where the data are evenly distributed over $0<t_{i}<10$. For $P$ values tested close to 2 , the phases of all these $0<t_{i}<1$ values would be within 0.5 in phase. This effect would yield larger simulated $z(f)$ values than the statistics based on $H_{0}$ do predict. In conclusion, erosion altered statistics and this had to be included into the simulations.

In most of the simulations, the "real" period was detected more often than the "false" periods. Only for $S=2 / 3$ and $\sigma_{P}=0.3$, the detection "false" periods was more likely. As expected, the probability of detecting the "real" period always increased when $n$ increased. There were clear cases, where the "real" period began to dominate as the sample size increased, and this lowered the probability of detecting "false" periods. In 
Table 9. Simulation results for the partly periodic cases $(S=2 / 3)$ for the rounded data, otherwise as in Table 4.

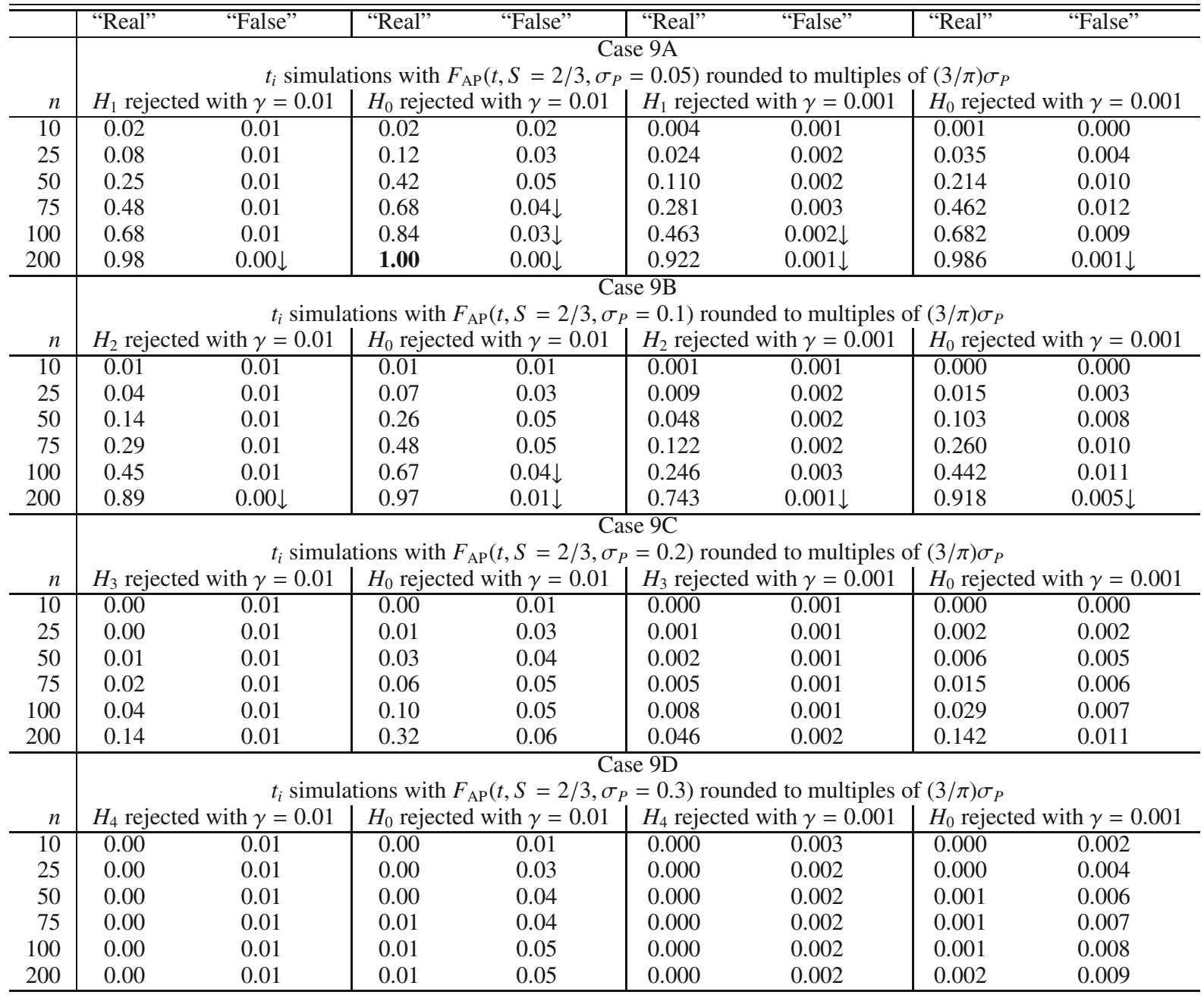

most cases, the probability of detecting "false" periods either increased or decreased monotonically when $n$ increased. In some cases, however, the probability of detecting "false" periods first increased, but then began to decrease. Such cases were marked with " $\downarrow$ " in Tables 4-9. In many such cases, this trend did lead to a "reliable" detection with larger sample sizes (e.g. Case $4 \mathrm{C}$ of Table 4). But there were also " $\downarrow$ " cases, where "reliable" detection was not achieved even with $n=200$ (e.g. $H_{1}$ was not rejected with $\gamma=0.01$ in Case $6 \mathrm{~A}$ of Table 6). Thus the notation " $\downarrow$ " in Tables 4-9 indicates that the sample size has increased so much that the "real" period has began to dominate over the "false" periods, but sometimes this effect was not yet strong enough to induce a "reliable" detection even with $n=200$.

Comparison of pairs of tables (i.e. Tables 4 and 7, Tables 5 and 8, Tables 6 and 9) revealed the effects of rounding. Rounding reduced the probability of detecting the "real" periodicity. As expected, this effect was strongest when the data were rounded with $\sigma_{P}=0.3$. On the other hand, rounding with $\sigma_{P}=0.05$ or 0.10 caused only a minor reduction in the probability of detecting "real" periodicity. There were only three cases, where rounding prevented "reliable" detection (i.e. $n=75$ in Case 7C of Table 7, $n=50$ in Case 8B of Table 8 and $n=200$ in Case 8C of Table 8). Rounding had no strong influence to the probability of "false" detections. But as already noted, the tested frequency range "conveniently" excluded frequencies like $f=3.33$ connected to rounding with $\sigma_{P}=0.3$. Furthermore, the Rayleigh test was sensitive only to unimodal phase distributions. The detection of the multimodal phase distributions induced by rounding would have been possible with other nonparametric methods, such as applied in Jetsu (1997) or Jetsu \& Pelt (2000).

In order to estimate the improvement in the quality and quantity of the impact crater data in recent years, we compared the data in our Table 1 to the data in Jetsu (1997), which are presented in our Table 10. The sample size $n$ of criterion $C_{2}$ has decreased from 34 to 33, while that of criterion $C_{3}$ has increased from 35 to 40 . The $P_{K}=\left(t_{n}-t_{1}\right) / K$ values of the old and new subsamples were calculated with $K=10$. The accuracy $\sigma_{P^{\prime}}$ of the data had improved: from 0.17 to 0.11 in $C_{2}$ and from 0.20 to 0.17 in $C_{3}$.

The main aim of this study was to determine the quantity $(n)$ and quality $\left(\sigma_{P}\right)$ of terrestrial impact crater data that would allow certain detection of periodicity - if present. Such "reliable" detections were listed in Tables 4-9 (numbers marked in bold). There were four Cases (A-D) in each of these six tables, where the results were determined for two preassigned significance levels $\gamma=0.01$ and 0.001 and for six different $n$ values, i.e. results were determined for $4 \times 6 \times 2 \times 6=288$ different alternatives. These "reliable" detections did not depend strongly on the chosen hypothesis, i.e. testing of $H_{0}$ versus $H_{1}, H_{2}, H_{3}$ or $H_{4}$. In only 5 out of all 288 alternatives, there were minor differences. These were the Cases 4C $(\gamma=0.01, n=75), 5 \mathrm{~B}(\gamma=0.01$, $n=50), 6 \mathrm{~A}(\gamma=0.01, n=200), 8 \mathrm{C}(\gamma=0.01, n=200)$ and 
Table 10. The data subsamples $C_{2}(=34)$ and $C_{3}(n=35)$ selected from the terrestrial impact crater data in Jetsu (1997). The selection criteria as well as all notations, are the same as in Table 1 .

\begin{tabular}{|c|c|c|c|c|c|c|c|}
\hline Crater & Location & $\overline{t_{i} \pm \sigma_{i}}$ & $\overline{D_{i}}$ & $C_{2}$ & $C_{3}$ & $k_{i}$ in $C_{2}$ & $k_{i}$ in $C_{3}$ \\
\hline Zhamanshin & Kazakhstan & $0.9 \pm 0.1$ & 13.5 & Yes & No & 1 & - \\
\hline Bosumtwi & Ghana & $1.03 \pm 0.02$ & 10.5 & Yes & No & 1 & - \\
\hline El'gygytgyn & Russia & $3.5 \pm 0.5$ & 18 & Yes & No & 1 & - \\
\hline Bigach & Kazakhstan & $6 \pm 3$ & 7 & Yes & Yes & 1 & 1 \\
\hline Shunak & Kazakhstan & $12 \pm 5$ & 3.1 & No & Yes & - & 1 \\
\hline Ries/Steinheim $\star$ & Germany/Germany & $15.0 \pm 0.7$ & 24.3 & Yes & Yes & 1 & 1 \\
\hline Haughton & Canada & $23 \pm 1$ & 24 & Yes & Yes & 1 & 1 \\
\hline Logancha & Russia & $25 \pm 20$ & 20 & Yes & Yes & 1 & 1 \\
\hline Popigai & Russia & $35 \pm 5$ & 100 & Yes & Yes & 2 & 2 \\
\hline Chesapeake Bay & USA & $35.5 \pm 0.6$ & 85 & Yes & Yes & 2 & 2 \\
\hline Wanapitei & Canada & $37 \pm 2$ & 7.5 & Yes & Yes & 2 & 2 \\
\hline Mistastin & Canada & $38 \pm 4$ & 28 & Yes & Yes & 2 & 2 \\
\hline Logoisk & Belarus & $40 \pm 5$ & 17 & Yes & Yes & 2 & 2 \\
\hline Chiyli & Kazakhstan & $46 \pm 7$ & 5.5 & Yes & Yes & 2 & 2 \\
\hline 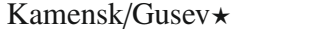 & Russia/Russia & $49.00 \pm 0.14$ & 25.2 & Yes & Yes & 2 & 2 \\
\hline Montagnais & Canada & $50.50 \pm 0.76$ & 45 & Yes & Yes & 3 & 2 \\
\hline Ragozinka & Russia & $55 \pm 5$ & 9 & Yes & Yes & 3 & 2 \\
\hline Marquez & USA & $58 \pm 2$ & 13 & Yes & Yes & 3 & 2 \\
\hline Chicxulub & Mexico & $64.98 \pm 0.05$ & 170 & Yes & Yes & 3 & 3 \\
\hline Kara/Ust-Kara $\star$ & Russia/Russia & $73.0 \pm 2.1$ & 69.6 & Yes & Yes & 3 & 3 \\
\hline Manson & USA & $73.8 \pm 0.3$ & 35 & Yes & Yes & 3 & 3 \\
\hline Lappajärvi & Finland & $77.3 \pm 0.4$ & 23 & Yes & Yes & 4 & 3 \\
\hline Boltysh & Ukraine & $88 \pm 3$ & 24 & Yes & Yes & 4 & 3 \\
\hline Dellen & Sweden & $89.0 \pm 2.7$ & 19 & Yes & Yes & 4 & 3 \\
\hline Steen River & Canada & $95 \pm 7$ & 25 & Yes & Yes & 4 & 4 \\
\hline Carswell/Zapadnaya & Canada/Ukraine & $115.0 \pm 7.1$ & 39.2 & Yes & Yes & 5 & 4 \\
\hline Zeleny Gai & Ukraine & $120 \pm 20$ & 2.5 & No & Yes & - & 5 \\
\hline Mien & Sweden & $121.0 \pm 2.3$ & 9 & Yes & Yes & 5 & 5 \\
\hline Tookoonooka & Australia & $128 \pm 5$ & 55 & Yes & Yes & 6 & 5 \\
\hline Romistrovka & Ukraine & $140 \pm 20$ & 2.7 & No & Yes & - & 5 \\
\hline Gosses Bluff & Australia & $142.5 \pm 0.5$ & 22 & Yes & Yes & 6 & 5 \\
\hline Mjølnir & Norway & $143 \pm 20$ & 40 & Yes & Yes & 6 & 5 \\
\hline Puchenzh-Katunki & Russia & $175 \pm 3$ & 80 & Yes & Yes & 8 & 6 \\
\hline Rochechouart & France & $186 \pm 8$ & 23 & Yes & Yes & 8 & 7 \\
\hline Wells Creek/Red Wing $\star$ & USA/USA & $200 \pm 18$ & 15 & Yes & Yes & 9 & 7 \\
\hline Manicouagan & Canada & $214 \pm 1$ & 100 & Yes & Yes & 9 & 8 \\
\hline Araguainha Dome & Brazil & $247.0 \pm 5.5$ & 40 & Yes & Yes & 10 & 9 \\
\hline 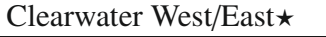 & Canada/Canada & $290 \pm 14$ & 44.4 & No & Yes & - & 10 \\
\hline
\end{tabular}

Table 11. Compilation of the results for simulations using the hypotheses $H_{1}, H_{2}, H_{3}$, or $H_{4}$.

\begin{tabular}{ll|cccc|ccccc}
\hline \hline & & $\sigma_{P}=0.05$ & $\sigma_{P}=0.1$ & $\sigma_{P}=0.2$ & $\sigma_{P}=0.3$ & $\sigma_{P}=0.05$ & $\sigma_{P}=0.1$ & $\sigma_{P}=0.2$ & $\sigma_{P}=0.3$ \\
& & $H_{1}$ & $H_{2}$ & $H_{3}$ & $H_{4}$ & $H_{1}$ & $H_{2}$ & $H_{3}$ & $H_{4}$ \\
& & & Rejected with $\gamma=0.01$ & & & & Rejected with $\gamma=0.001$ & \\
\hline Table 4: & $S=0$ & $n \geq 10$ & $n \geq 25$ & $n \geq 100$ & $\dagger$ & $n \geq 25$ & $n \geq 25$ & $n \geq 200$ & $\dagger$ \\
Table 5: & $S=1 / 3$ & $n \geq 50$ & $n \geq 75$ & $n \geq 200$ & $\dagger$ & $n \geq 75$ & $n \geq 100$ & $\downarrow$ & $\dagger$ \\
Table 6: & $S=2 / 3$ & $\downarrow$ & $\downarrow$ & $\dagger$ & $\dagger$ & $\downarrow$ & $\downarrow$ & $\dagger$ \\
Table 7: & $S=0$ and $t_{\mathrm{i}}$ rounded & $n \geq 10$ & $n \geq 25$ & $n \geq 100$ & $\dagger$ & $n \geq 25$ & $n \geq 25$ & $n \geq 200$ & $\dagger$ \\
Table 8: & $S=1 / 3$ and $t_{\mathrm{i}}$ rounded & $n \geq 50$ & $n \geq 75$ & $\downarrow$ & $\dagger$ & $n \geq 75$ & $n \geq 100$ & $\downarrow$ & $\dagger$ \\
Table 9: & $S=2 / 3$ and $t_{\mathrm{i}}$ rounded & $\downarrow$ & $\downarrow$ & $\dagger$ & $\dagger$ & $\downarrow$ & $\downarrow$ & $\dagger$ & $\dagger$ \\
\hline
\end{tabular}

9A $(\gamma=0.01, n=200)$. But even in these cases, the "real" period was emerging (i.e. marked with $\downarrow$ ) regardless of the chosen hypothesis, except for the Case 5B $(\gamma=0.01, n=50)$. As already discussed, the statistics based on $H_{0}$ were found to be less reliable. Therefore we shall hereafter concentrate only on the results based on $\mathrm{H}_{1}, \mathrm{H}_{2}, \mathrm{H}_{3}$, or $\mathrm{H}_{4}$. These results for "reliable" detections have been compiled into Table 11, which shows the lowest number $n$ of simulated time points $t_{i}$, that was needed for "reliable" detection of the "real" periodicity with the preassigned significance levels of $\gamma=0.01$ or 0.001 . The symbol " $\downarrow$ " is used to denote the cases where the "real" period was emerging with $n=200$, but the detection was not yet "reliable". The cases where "reliable" detection would require $n>200$ have been denoted with " $\uparrow$ ".

\section{Conclusions}

If all impacts were periodic ( $S=0)$, "reliable" detection of periodicity would be possible for numerous combinations, where $\sigma_{P} \leq 0.2$ and $n \leq 200$ (Table 11). In fact, very accurate data 
having $\sigma_{P}=0.05$ would yield "reliable" detections even for small samples like $n=10(\gamma=0.01)$ or $n=25(\gamma=0.001)$. If this accuracy deteriorates to $\sigma_{P} \geq 0.3$, "reliable" detections become impossible even if all impacts were periodic and $n=200$.

If one third of the impacts were aperiodic $(S=1 / 3)$, "reliable" detections would require from three to five times larger samples $(n \geq 50)$ than in the fully periodic case $(S=0)$. Furthermore, the accuracy would have to be at least $\sigma_{P} \leq 0.1$.

The results for $S=2 / 3$ were, mildly put, discouraging. There was no chance of "reliable" detection even with $\sigma_{P}=0.05$ and $n=200$. One may find indications of periodicity only in very large $(n \geq 100)$ and very accurate $\left(\sigma_{P} \leq 0.1\right)$ samples (Table 11: $S=2 / 3$ cases marked with $\downarrow$ ). In other words, if two thirds of all impacts are caused by asteroids, then real periodicity due to comet impacts could not be reliably detected (even if present).

Currently, the ratio between periodic and aperiodic impacts is unknown. For example, Bailey \& Stagg (1988) have argued that $98 \%$ of terrestrial impact craters have been produced by Earth-crossing asteroids. Bottke et al. (2002b) have estimated that approximately $6 \%$ of NEOs are comets originating in the Jupiter-family comet region, and the rest are asteroids from different regions of the main asteroid belt. All asteroid impacts on Earth have most probably been aperiodic and they seem to dominate the possibly periodic comet impacts. Moreover, there are no direct evidence of any terrestrial comet showers and there are no reasons for assuming that all comet impacts would have been periodic. In the presence of an aperiodic part, "reliable" detection is possible only with very accurate and large samples. Comparison of Tables 1 and 10 in Sect. 6 revealed only minor quantity and quality improvements during the past decade, i.e. between the years 1997 and 2008. Hence a "reliable" detection is highly unlikely in the near future.

Our main conclusion is: "reliable" detection of "real" periodicity from the currently available terrestrial impact crater data is impossible - unless all impacts have been due to periodic comet showers.
Acknowledgements. The authors wish to thank Professors Philippe Claeys and Karri Muinonen for valuable comments on the manuscript.

\section{References}

Alvarez, W., \& Muller, R. A. 1984, Nature, 308, 718 Bailey, M. E., \& Stagg, C. R. 1988, MNRAS, 235, 1

Bottke, W. F., Vokrouhlicky, Jr., D., Rubincam, D. P., \& Broz, M. 2002, Asteroids III (Tucson: University of Arizona Press), 395

Bottke, W. F., Morbidelli, A., Jedicke, R., et al. 2002, Icarus, 156, 399

Brazier, K. T. S. 1994, MNRAS, 268, 709

Chang, H.-Y., \& Moon, H.-K. 2005, PASJ, 57, 487

Davis, M., Hut, P., \& Muller, R. A. 1984, Nature, 308, 715

Deutsch, A., \& Schaerer, U. 1994, Meteoritics, 29, 301

Farley, K. A. 1998, Planet. Rep., 18, 197, 9

Gladman, B. J., Migliorini, F., Morbidelli, A., et al. 1997, Science, 277, 197

Grieve, R. A. F., \& Pesonen, L. J. 1996, Earth, Moon and Planets, 72, 357

Grieve, R. A. F., Sharpton, V. L., Rupert, J. D., \& Goodacre, A. K. 1988, Lunar Plan. Sci. Conf. 18, ed. G. Ryder, 375

Hills, J. G. 1981, AJ, 86, 1730

Jetsu, L. 1997, A\&A, 321, L33

Jetsu, L., \& Pelt, J. 2000, A\&A, 353, 409

Kelley, S. P. 2003, EGS - AGU - EUG Joint Assembly, Abstracts from the meeting held in Nice, France, 6-11 April, abstract 13907

Kelley, S. P. 2003, American Geophysical Union, Fall Meeting, abstract V22E04

Kruger, A. T., Loredo, T. J., \& Wasserman, I. 2002, ApJ, 576, 932

Moon, H. K., Min, B. H., \& Kim, S. L. 2003, JASS, 20, 269

Neukum, G., \& Ivanov, B. A. 1994, Hazards Due to Comets and Asteroids, ed. T. Gehrels, M. S. Matthews, \& A. M. Schumann (Tucson: University of Arizona Press), 359

Rampino, M. R., \& Stothers, R. B. 1984, Nature, 308, 709

Schwartz, R. D., \& James, P. B. 1984, Nature, 308, 712

Shoemaker, E. M., Wolfe, R. F., \& Shoemaker, C. S. 1990, Geol. Soc. Am. Spec. Pap., 247, 155

Stothers, R. B. 1998, MNRAS, 300, 1098

Stothers, R. B. 2006, MNRAS, 365, 178

Tagle, R., \& Claeys, P. 2006, Science, 305, 492

Vokrouhlicky, D., \& Farinella, P. 2000, Nature, 407, 606

Whitmire, D. P., \& Jackson, A. A. 1984, Nature, 308, 713

Wickramasinghe, J. T., \& Napier, W. M. 2008, MNRAS, 387, 153

Yabushita, S. 2004, MNRAS, 355, 51 